\title{
A Comparative Study of Ion Chemistry of Groundwater Samples of Typical Highland and Midland Sub-watersheds of the Manimala River Basin, Kerala, South India
}

\author{
Vadakkepurakkal Balakrishnan Rekha ${ }^{1}$, Aikara Varkey George ${ }^{2}$ and Mendaz \\ Rita $^{3}$ \\ ${ }^{1}$ Department of Environmental Science, Central University of Kerala, Kasaragode, Kerala, India \\ ${ }^{2}$ Mahatma Gandhi University, Kottayam, Kerala, India \\ ${ }^{3}$ Department of Chemistry, Govt Engineering College, Thrissur, Kerala, India
}

cross $^{\text {ref }}$ http://dx.doi.org/10.5755/j01.erem.66.4.4037

(Received in September, 2013; accepted in December, 2013)

\begin{abstract}
Results of the detailed study of the chemistry of ions present in the groundwater samples of Peruvanthanam and Valiyathodu sub-watersheds of the Manimala river basin are analysed using the AQUACHEM 4.0 software to understand general chemical characteristics and geochemical processes involved. The study reveals that cations, such as sodium $\left(\mathrm{Na}^{+}\right)$, potassium $\left(\mathrm{K}^{+}\right)$, calcium $\left(\mathrm{Ca}^{2+}\right)$, and magnesium $\left(\mathrm{Mg}^{2+}\right)$ and anions, such as bicarbonate $\left(\mathrm{HCO}_{3}^{-}\right)$, sulphate $\left(\mathrm{SO}_{4}^{2-}\right)$, chloride $\left(\mathrm{Cl}^{-}\right)$, nitrate $\left(\mathrm{NO}_{3}^{-}\right)$ are present. Recognition methods such as (a) Box and Whisker diagram (b) Piper diagram (c) Durov diagram (d) Radial plot and (e) Stiff diagram were prepared to delineate the seasonal variations in chemical constituents. The major ionic concentration of the groundwater samples of Peruvanthanam sub-watershed is $\mathrm{Mg}^{2+}>\mathrm{Ca}^{2+}>\mathrm{HCO}_{3}^{-}>\mathrm{Cl}^{-}>\mathrm{SO}_{4}{ }^{2-}>\mathrm{NO}_{3}^{-}>\mathrm{Na}^{+}>\mathrm{K}^{+}$and that of Valiyathodu is $\mathrm{Mg}^{2+}>\mathrm{Ca}^{2+}>\mathrm{HCO}_{3}>\mathrm{Cl}^{-}>\mathrm{NO}_{3}{ }^{-}$ $>\mathrm{SO}_{4}{ }^{2-}>\mathrm{Na}^{+}>\mathrm{K}^{+}$. A critical analysis of Piper diagram reveals that in Peruvanthanam sub-watershed the pre monsoon groundwater samples belong to the zone of bicarbonates, chloride and sulphates, the monsoon season samples belong to the bicarbonate and chloride zone and the post monsoon samples belong to the zone of prevailing bicarbonates. In Valiyathodu sub-watershed, the pre monsoon and post monsoon samples have dominant bicarbonates, while the monsoon samples show predominance of both bicarbonates and chloride. Radial plots and Stiff diagrams for Peruvanthanam sub-watershed show that $\mathrm{Mg}^{2+}-\mathrm{Ca}^{2+}-\mathrm{HCO}_{3}{ }^{-} \mathrm{Mg}^{2+}-\mathrm{Ca}^{2+}$ $\mathrm{Cl}^{-}$and $\mathrm{Mg}^{2+}-\mathrm{Ca}^{2+}-\mathrm{HCO}_{3}{ }^{-}-\mathrm{Cl}^{-}$are the dominant water types during the pre monsoon season. $\mathrm{Mg}^{2+}-\mathrm{Ca}^{2+}{ }_{-}$ $\mathrm{HCO}_{3}^{-}-\mathrm{Cl}^{-}, \mathrm{Mg}^{2+}-\mathrm{Ca}^{2+}-\mathrm{Cl}^{-}-\mathrm{HCO}_{3}^{-}$and $\mathrm{Mg}^{2+}-\mathrm{Ca}^{2+}-\mathrm{HCO}_{3}^{-}-\mathrm{Cl}^{-}-\mathrm{SO}_{4}{ }^{2-}$ are dominant during the monsoon and $\mathrm{Mg}^{2+}-\mathrm{Ca}^{2+}-\mathrm{HCO}_{3}{ }^{-}$and $\mathrm{Mg}^{2+}-\mathrm{Ca}^{2+}-\mathrm{HCO}_{3}{ }^{-}-\mathrm{Cl}^{-}$are the dominant water types during the post monsoon season. In Valiyathodu sub-watershed during the pre monsoon $-\mathrm{Mg}^{2+}-\mathrm{Ca}^{2+}-\mathrm{HCO}_{3}{ }^{-}-\mathrm{Cl}^{-}$and $\mathrm{Mg}^{2+}-\mathrm{Ca}^{2+}-\mathrm{HCO}_{3}{ }^{-}$, during the monsoonm $-\mathrm{Mg}^{2+}-\mathrm{Ca}^{2+}-\mathrm{HCO}_{3}{ }_{-}-\mathrm{Cl}^{-}, \mathrm{Mg}^{2+}-\mathrm{Ca}^{2+}-\mathrm{Cl}^{-}-\mathrm{HCO}_{3}{ }^{-}$and $\mathrm{Ca}^{2+}-\mathrm{Mg}^{2+}-\mathrm{HCO}_{3}{ }^{-}-\mathrm{Cl}^{-}$, and during the post monsoon $-\mathrm{Mg}^{2+}-\mathrm{Ca}^{2+}-\mathrm{HCO}_{3}^{-}-\mathrm{Cl}^{-}$and $\mathrm{Ca}^{2+}-\mathrm{Mg}^{2+}-\mathrm{HCO}_{3}^{-}-\mathrm{Cl}^{-}$are the dominant water types.
\end{abstract}

Keywords: the Manimala, ion chemistry, Piper diagram, Peruvanthanam sub-watershed, Valiyathodu sub-watershed

\section{Introduction}

Millions of people rely on groundwater that contains an excess of cations and anions (Ramesh et al. 2012). The character of groundwater in different aquifers over space and time proved to be an important technique in solving different geochemical problems (Chebotarev 1955, Hem 1959, Back and
Hanshaw 1965, Gibbs 1970, Srinivasamoorthy 2005). Due to interactions with the atmosphere, surface environment, nature of soil and bedrock a wide range of different elements can dissolve in groundwater (Subramanian 2000). The solute load of water determines the total dissolved solids which in turn 
affect the quality of water. It is a very useful indicator of geological evolution, mode of origin within hydrological cycles, soil or rock mass influences, the extent of pollution and contamination etc. Usually groundwater has much higher concentrations of most constituents as it has been in contact with rock for longer time than shallow or young water. The present study is carried out to understand the concentration and distribution of both cations and anions of Peruvanthanam and Valiyathodu sub-watersheds during different seasons.

The areas selected for this investigation i.e. Peruvanthanam and Valiyathodu sub-watersheds belong to typical highland and midland subwatersheds of the Manimala river basin. Peruvanthanam sub-watershed is structurally not complex and is characterised by high run off and low infiltration, whereas Valiyathodu is structurally more complex and is characterised by low run off and high infiltration. Peruvanthanam sub-watershed (Fig.1) lies between $9^{0} 29^{\prime} 00^{\prime \prime}$ to $9^{0} 34^{\prime} 00^{\prime \prime} \mathrm{N}$ latitude and $76^{0} 53^{\prime} 00^{\prime \prime}$ to $76^{0} 59^{\prime} 00^{\prime \prime}$ E longitude and Valiyathodu subwatershed (Fig. 2) lies between $9^{0} 30^{\prime} 00^{\prime \prime}$ to $9^{0} 37^{\prime} 00^{\prime \prime}$ $\mathrm{N}$ latitude and $76^{\circ} 41^{\prime} 00^{\prime \prime}$ to $76^{\circ} 46^{\prime} 00^{\prime \prime} \mathrm{E}$ longitude. Peruvanthanam sub-watershed covers the area of $56.42 \mathrm{~km}^{2}$ and Valiyathodu $-54.85 \mathrm{~km}^{2}$.

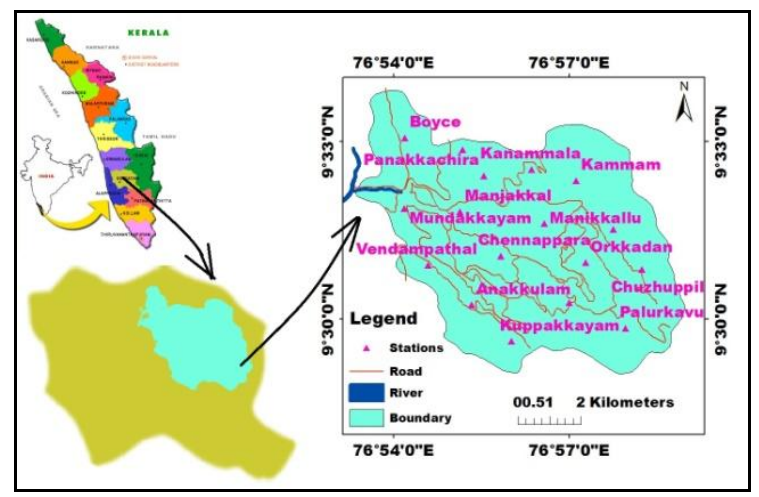

Fig. 1. Location map of Peruvanthanam sub-watershed

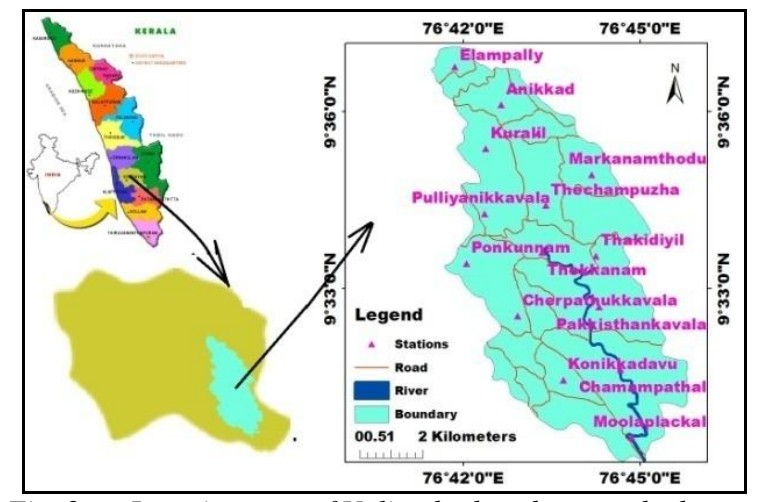

Fig. 2. Location map of Valiyathodu sub-watershed

\section{Methods}

In this study, 17 and 15 groundwater samples were collected from Peruvanthanam and Valiyathodu sub-watersheds respectively for pre monsoon, monsoon and post monsoon seasons, and chemical analysis of both cations and anions were carried out as per the methodology prescribed by APHA (1989). It is convenient to divide the dissolved constituents into major components i.e. the predominant cations and anions. Sodium $\left(\mathrm{Na}^{+}\right)$, potassium $\left(\mathrm{K}^{+}\right)$, calcium $\left(\mathrm{Ca}^{2+}\right)$ and magnesium $\left(\mathrm{Mg}^{2+}\right)$ are dominant cations and bicarbonate $\left(\mathrm{HCO}_{3}^{-}\right)$, sulphate $\left(\mathrm{SO}_{4}{ }^{2-}\right)$, chloride $\left(\mathrm{Cl}^{-}\right)$ and nitrate $\left(\mathrm{NO}_{3}{ }^{-}\right)$are dominant anions; these ions were added as attribute data to the AQUACHEM 4.0 software. The study illustrates the use of recognition methods such as (a) Box and Whisker diagram, (b) Piper diagram, (c) Durov diagram, (d) Radial plot and (e) Stiff diagram to delineate seasonal variations in chemical constituents, and thereby to point out the source contributions of vital major ions in the groundwater resources under various geomorphic conditions of both sub-watersheds. Here the chemistry of ions is depicted in different ways using the AQUACHEM 4.0 software to understand general chemical characteristics of groundwater and geochemical processes that are involved in chemical evolution of water that flows through Peruvanthanam and Valiyathodu sub-watersheds.

\section{Results and Discussion}

\subsection{Box and Whisker diagram or Box plot}

Box and Whisker diagram or Box plot shows the distribution of major ions in the ground water samples during the study period. It represents an inter quartile range and either end of the line indicates the minimum and maximum values. Box plot like other visual methods is more than a substitute for a table. It is a tool that can improve our reasoning about quantitative information (Williamson 1989). In descriptive statistics, Box plot is a convenient way of graphical depicting of the groups of numerical data through their five number summaries: the smallest observation (sample minimum), lower quartile (Q1), median (Q2), upper quartile (Q3) and largest observation (sample maximum). Box plot displays differences between populations without making any assumptions of the underlying statistical distribution. The spacing between the different parts of the box helps indicate the degree of dispersion and skewed data. It can be drawn either horizontally or vertically. Distribution of major ions in the groundwater samples of both Peruvanthanam and Valiyathodu subwatersheds are represented in Figs 3 and 4. The box represents the inter quartile range and either end of the line indicates the minimum and maximum values. The major ionic concentration of the groundwater samples of Peruvanthanam subwatershed is $\mathrm{Mg}^{2+}>\mathrm{Ca}^{2+}>\mathrm{HCO}_{3}{ }^{-}>\mathrm{Cl}^{-}>\mathrm{SO}_{4}{ }^{2-}>\mathrm{NO}_{3}{ }^{-}>\mathrm{Na}^{+}>\mathrm{K}^{+}$and that of Valiyathodu sub-watershed is $\mathrm{Mg}^{2+}>\mathrm{Ca}^{2+}>\mathrm{HCO}_{3}^{-}>\mathrm{Cl}^{-}>\mathrm{NO}_{3}{ }^{-}>\mathrm{SO}_{4}{ }^{2-}>\mathrm{Na}^{+}>\mathrm{K}^{+}$. In Valiyathodusub-watershed, $\mathrm{NO}_{3}{ }^{-}$is greater than $\mathrm{SO}_{4}{ }^{2-}$. 


\subsection{Piper diagram}

Piper diagram is an excellent tool for hydro chemical analysis using a series of water quality data in a spatial context (Piper 1944). It has become a widespread method for understanding and describing chemical evolution of groundwater which depends on pattern recognition techniques and permits the classification of water. It can also define the patterns of spatial change in the water chemistry among geological units, along a line of the section or along a path line (Domenico and Schwartz 1998). It is therefore useful in understanding the water flow and water quality (Back 1960, Ophori and Toth 1989, Sikdar et.al 1993, Sikdar and Bhattacharya 1999), and the changes in water types and mixing relationships based on the relative proportions of major ions rather than the bulk concentrations. Hence, it is the most widely used method for delineation of hydrochemical evolution and identification of dominant processes that control water chemistry. Piper diagrams of both Peruvanthanam and Valiyathodu subwatersheds are shown in Figs 5 and 6. Groundwater samples of Peruvanthanam sub-watershed depicted in Fig. 5 are of normal earth alkaline origin. In groundwater samples that come under zone ' $a$ ' bicarbonates prevail. Compared to the other two seasons, post monsoon groundwater samples are dominant in this zone. The samples that come under zone ' $b$ ' contain bicarbonates, chloride and sulphates. Pre monsoon groundwater samples are dominant in this zone. In zone 'c' bicarbonates and chlorides dominate.. Only groundwater samples of the monsoon season come under this zone. Piper diagram of Valiyathodu subwatershed, i.e. Fig. 6 , reveals that the groundwater samples under zone ' $a$ ' are predominantly bicarbonates. The pre monsoon and post monsoon samples are dominant in this zone. The samples under zone ' $b$ ' contain bicarbonates, chloride and sulphates. The pre monsoon, monsoon and post monsoon groundwater samples come under this zone. In zone 'c' bicarbonates and chlorides dominate. Only the groundwater samples of the monsoon season come under this zone.

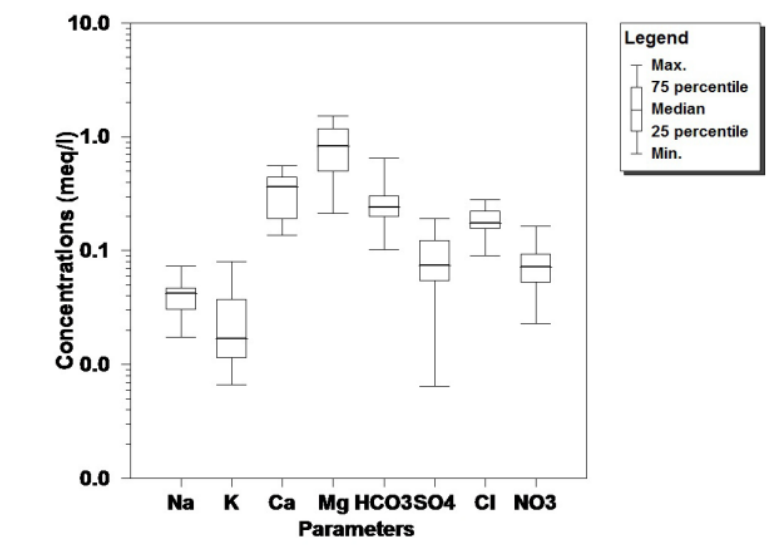

Fig. 3. Box plot showing ion distribution in the groundwater samples of Peruvanthanam subwatershed
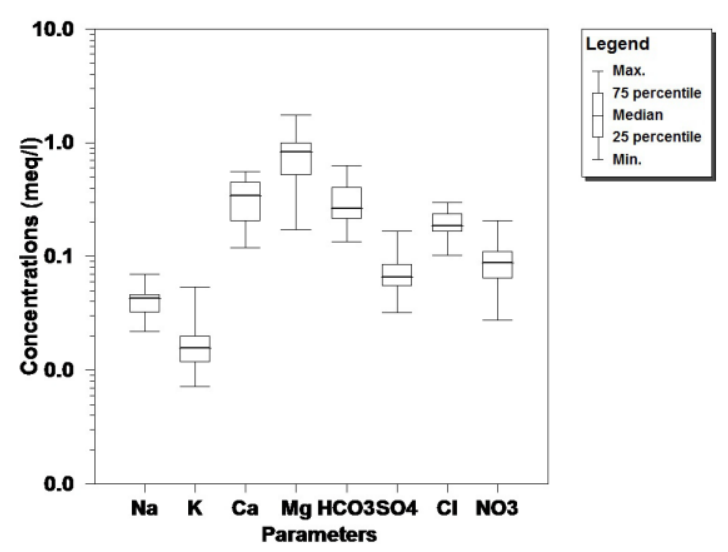

Fig. 4. Box plot showing ion distribution in the groundwater samples of Valiyathodu subwatershed

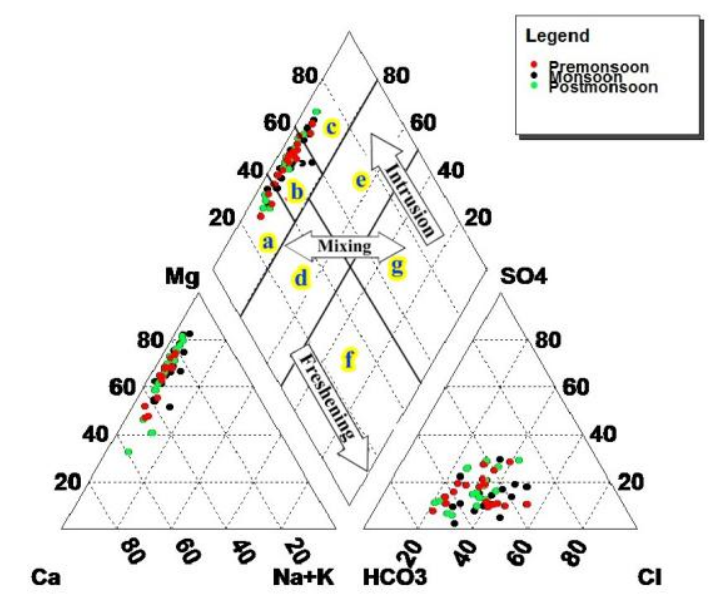

Fig. 5. Piper diagram showing the water type of Peruvanthanam groundwater samples of subwatershed

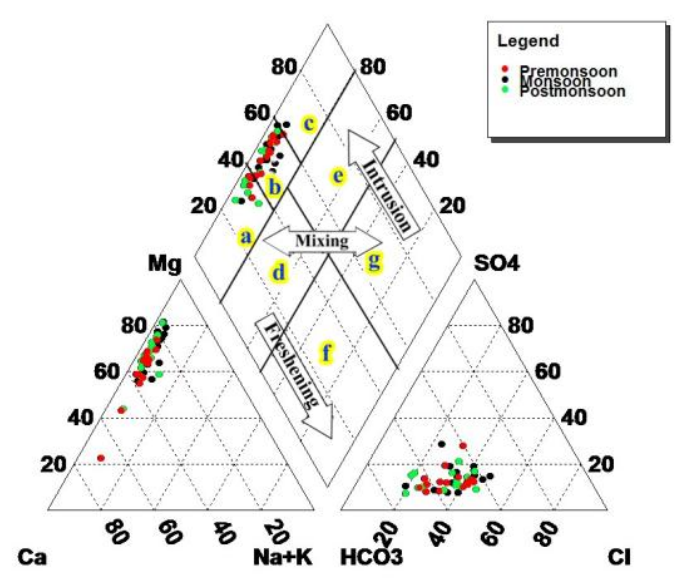

Fig. 6. Piper diagram showing the water type of groundwater samples of Valiyathodus subwatershed

\subsection{Durov diagram}

The advantage of Durov diagram is that it displays some possible geochemical processes that could affect the water genesis (Durov 1948). Durov diagram of Peruvanthanm sub-watershed (Fig. 7) 
depicts that groundwater samples fall in fields $1,2,4$ and 5. In field $1, \mathrm{HCO}_{3}{ }^{-}$and $\mathrm{Ca}^{2+}$ are dominant which indicates recharging of water in limestone and sandstone aquifers. Pre monsoon groundwater samples are dominant in field 1 . In field 2, the water type is dominated by $\mathrm{Ca}^{2+}, \mathrm{Mg}^{2+}$ and $\mathrm{HCO}_{3}^{-}$ions. If $\mathrm{Mg}^{2+}$ is significant, the association with dolomite is presumed, and if $\mathrm{Na}^{+}$is significant, an important ion exchange is presumed. Few samples come under this field. In field $4 \mathrm{SO}_{4}{ }^{2-}$ is dominant. Few groundwater samples come under this field and they are post monsoon samples. In field 5 no dominant anion or cation are observed which indicates water exhibiting simple dissolution or mixing towards $\mathrm{Cl}^{-}$. Pre monsoon samples are dominant in this zone. In Valiyathodu sub-watershed (Fig. 8), in field $1 \mathrm{HCO}_{3}{ }^{-}$ and $\mathrm{Ca}^{2+}$ are dominant, indicating recharge of water in limestone and sandstone aquifers. In field 2 the water type is dominated by $\mathrm{Ca}^{2+}, \mathrm{Mg}^{2+}$ and $\mathrm{HCO}_{3}{ }^{-}$ions. If $\mathrm{Mg}^{2+}$ is significant, association with dolomite is presumed, and if $\mathrm{Na}+$ is significant, an important ion exchange is presumed. In field $4 \mathrm{SO}_{4}{ }^{2-}$ is dominant. Few groundwater samples come under this field. In field 5 the absence of dominant anions or cations indicates water exhibiting simple dissolution or mixing towards $\mathrm{Cl}^{-}$.

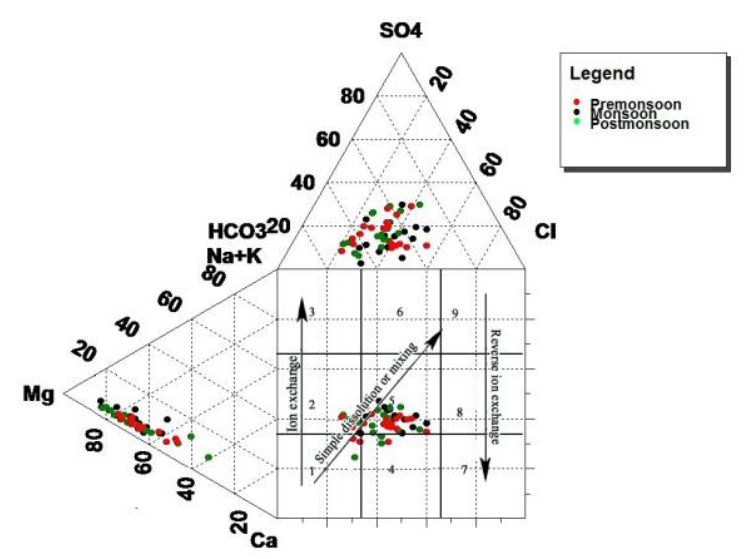

Fig. 7. Durov diagram showing processes of dissolution and mixing in groundwater samples of Peruvanthanam sub-watershed

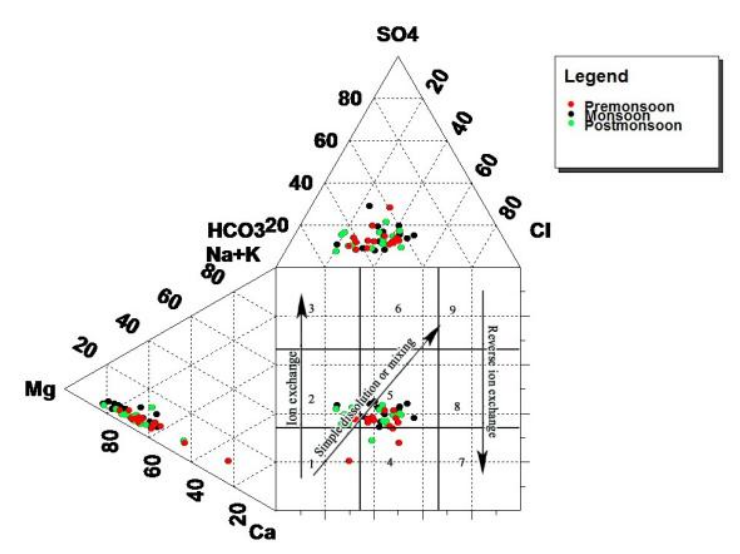

Fig. 8. Durov diagram showing processes of dissolution and mixing in groundwater samples of Valiyathodu sub-watershed

\subsection{Radial plots}

A radial plot is a graphical display for comparing the estimates that have differing precisions. It is a scatter plot of standardised estimates against reciprocals of standard errors, possibly with respect to a transformed scale, designed so that the original estimates can be compared and interpreted. The estimates may be means, regression coefficients, proportions, rates, odd ratios, random effects, or indeed any parameter estimates that merit the comparison between individuals or groups. The water types of each well of both Peruvanthanam and Valiyathodu sub-watersheds are represented in Table 1 and Table 2 respectively.

Table 1. Water types of Peruvanthanam sub-watershed

\begin{tabular}{|c|c|c|c|}
\hline $\mathbf{N}$ & $\begin{array}{c}\text { Pre } \\
\text { monsoon }\end{array}$ & Monsoon & $\begin{array}{c}\text { Post } \\
\text { monsoon }\end{array}$ \\
\hline P1 & $\begin{array}{l}\mathrm{Mg}^{2+}-\mathrm{Ca}^{2+}- \\
\mathrm{HCO}_{3}^{-}-\mathrm{Cl}^{-}\end{array}$ & $\begin{array}{c}\mathrm{Mg}^{2+}-\mathrm{Ca}^{2+}-\mathrm{Cl}^{-}- \\
\mathrm{HCO}_{3}^{-}\end{array}$ & $\begin{array}{c}\mathrm{Mg}^{2+}-\mathrm{Ca}^{2+}- \\
\mathrm{HCO}_{3}^{-}\end{array}$ \\
\hline $\mathrm{P} 2$ & $\begin{array}{c}\mathrm{Mg}^{2+}-\mathrm{Ca}^{2+}- \\
\mathrm{Cl}^{-}\end{array}$ & $\begin{array}{c}\mathrm{Mg}^{2+}-\mathrm{Ca}^{2+}- \\
\mathrm{HCO}_{3}^{-}\end{array}$ & $\begin{array}{l}\mathrm{Mg}^{2+}-\mathrm{Ca}^{2+}- \\
\mathrm{Cl}^{-}\end{array}$ \\
\hline $\mathrm{P} 3$ & $\begin{array}{l}\mathrm{Mg}^{2+}-\mathrm{Ca}^{2+}- \\
\mathrm{HCO}_{3}^{-}-\mathrm{Cl}^{-}\end{array}$ & $\mathrm{Mg}^{2+}-\mathrm{Ca}^{2+}-\mathrm{Cl}^{-}$ & $\mathrm{Mg}^{2+}-\mathrm{Ca}^{2+}$ \\
\hline $\mathrm{P} 4$ & $\mathrm{Mg}^{2+}-\mathrm{Ca}^{2+}$ & $\begin{array}{l}\mathrm{Mg}^{2+}-\mathrm{Ca}^{2+}- \\
\mathrm{HCO}_{3}^{-}-\mathrm{Cl}^{-}\end{array}$ & $\begin{array}{l}\mathrm{Ca}^{2+}-\mathrm{Mg}^{2+}- \\
\mathrm{HCO}_{3}^{-}-\mathrm{Cl}^{-}\end{array}$ \\
\hline P5 & $\begin{array}{c}\mathrm{Mg}^{2+}-\mathrm{Ca}^{2+}- \\
\mathrm{HCO}_{3}^{-}\end{array}$ & $\begin{array}{l}\mathrm{Mg}^{2+}-\mathrm{Ca}^{2+}- \\
\mathrm{HCO}_{3}^{-}-\mathrm{Cl}^{-}\end{array}$ & $\mathrm{Mg}^{2+}-\mathrm{Ca}^{2+}$ \\
\hline P6 & $\begin{array}{l}\mathrm{Mg}^{2+}-\mathrm{Ca}^{2+}- \\
\mathrm{HCO}_{3}^{-}\end{array}$ & $\begin{array}{c}\mathrm{Mg}^{2+}-\mathrm{Ca}^{2+}- \\
\mathrm{HCO}_{3}^{-}-\mathrm{Cl}^{-}- \\
\mathrm{SO}_{4}^{2-}\end{array}$ & $\mathrm{Mg}^{2+}-\mathrm{Ca}^{2+}$ \\
\hline P7 & $\begin{array}{c}\mathrm{Mg}^{2+}-\mathrm{Ca}^{2+}- \\
\mathrm{Cl}^{-}\end{array}$ & $\begin{array}{l}\mathrm{Mg}^{2+}-\mathrm{Ca}^{2+}- \\
\mathrm{HCO}_{3}^{-}-\mathrm{Cl}^{-}\end{array}$ & $\begin{array}{l}\mathrm{Mg}^{2+}-\mathrm{Ca}^{2+}- \\
\mathrm{HCO}_{3}^{-}-\mathrm{Cl}^{-}\end{array}$ \\
\hline P8 & $\begin{array}{c}\mathrm{Mg}^{2+}-\mathrm{Ca}^{2+}- \\
\mathrm{HCO}_{3}{ }^{-}\end{array}$ & $\mathrm{Mg}^{2+}-\mathrm{HCO}_{3}^{-}$ & $\begin{array}{l}\mathrm{Ca}^{2+}-\mathrm{Mg}^{2+}- \\
\mathrm{HCO}_{3}^{-}-\mathrm{Cl}^{-}\end{array}$ \\
\hline P9 & $\begin{array}{c}\mathrm{Mg}^{2+}-\mathrm{Ca}^{2+}- \\
\mathrm{HCO}_{3}^{-}\end{array}$ & $\begin{array}{l}\mathrm{Mg}^{2+}-\mathrm{Ca}^{2+}- \\
\mathrm{HCO}_{3}^{-}-\mathrm{Cl}^{-}\end{array}$ & $\begin{array}{c}\mathrm{Mg}^{2+}-\mathrm{Ca}^{2+}- \\
\mathrm{HCO}_{3}^{-}\end{array}$ \\
\hline P10 & $\begin{array}{c}\mathrm{Mg}^{2+}-\mathrm{Ca}^{2+}- \\
\mathrm{HCO}_{3}{ }^{-}\end{array}$ & $\mathrm{Mg}^{2+}-\mathrm{HCO}_{3}{ }^{-}$ & $\begin{array}{c}\mathrm{Mg}^{2+}-\mathrm{Ca}^{2+}- \\
\mathrm{HCO}_{3}^{-}\end{array}$ \\
\hline P11 & $\begin{array}{l}\mathrm{Mg}^{2+}-\mathrm{Ca}^{2+}- \\
\mathrm{HCO}_{3}^{-}-\mathrm{Cl}^{-}\end{array}$ & $\begin{array}{c}\mathrm{Mg}^{2+}-\mathrm{Ca}^{2+}-\mathrm{Cl}^{-}- \\
\mathrm{HCO}_{3}^{-}\end{array}$ & $\begin{array}{c}\mathrm{Mg}^{2+}-\mathrm{Ca}^{2+}- \\
\mathrm{HCO}_{3}{ }^{-}\end{array}$ \\
\hline P12 & $\begin{array}{l}\mathrm{Mg}^{2+}-\mathrm{Ca}^{2+}- \\
\mathrm{HCO}_{3}^{-}-\mathrm{Cl}^{-}\end{array}$ & $\begin{array}{l}\mathrm{Mg}^{2+}-\mathrm{Ca}^{2+}- \\
\mathrm{HCO}_{3}^{-}-\mathrm{Cl}^{-}\end{array}$ & $\begin{array}{c}\mathrm{Mg}^{2+}-\mathrm{Ca}^{2+}- \\
\mathrm{HCO}_{3}^{-}\end{array}$ \\
\hline P13 & $\begin{array}{c}\mathrm{Mg}^{2+}-\mathrm{Ca}^{2+}- \\
\mathrm{HCO}_{3}^{-}\end{array}$ & $\begin{array}{c}\mathrm{Mg}^{2+}-\mathrm{Ca}^{2+}-\mathrm{Cl}^{-}- \\
\mathrm{HCO}_{3}^{-}\end{array}$ & $\begin{array}{c}\mathrm{Mg}^{2+}-\mathrm{Ca}^{2+}- \\
\mathrm{HCO}_{3}^{-}\end{array}$ \\
\hline P14 & $\begin{array}{l}\mathrm{Mg}^{2+}-\mathrm{Ca}^{2+}- \\
\mathrm{HCO}_{3}^{-}-\mathrm{Cl}^{-}\end{array}$ & $\begin{array}{l}\mathrm{Mg}^{2+}-\mathrm{Ca}^{2+}- \\
\mathrm{HCO}_{3}^{-}-\mathrm{Cl}^{-}\end{array}$ & $\begin{array}{l}\mathrm{Ca}^{2+}-\mathrm{Mg}^{2+}- \\
\mathrm{HCO}_{3}^{-}-\mathrm{Cl}^{-}\end{array}$ \\
\hline P15 & $\begin{array}{c}\mathrm{Mg}^{2+}-\mathrm{Ca}^{2+}- \\
\mathrm{HCO}_{3}{ }^{-}\end{array}$ & $\begin{array}{l}\mathrm{Mg}^{2+}-\mathrm{Ca}^{2+}- \\
\mathrm{HCO}_{3}^{-}-\mathrm{Cl}^{-}\end{array}$ & $\begin{array}{l}\mathrm{Mg}^{2+}-\mathrm{Ca}^{2+}- \\
\mathrm{HCO}_{3}{ }^{-}\end{array}$ \\
\hline P16 & $\begin{array}{c}\mathrm{Mg}^{2+}-\mathrm{Ca}^{2+}- \\
\mathrm{HCO}_{3}^{-}\end{array}$ & $\begin{array}{c}\mathrm{Mg}^{2+}-\mathrm{Ca}^{2+}- \\
\mathrm{HCO}_{3}^{-}\end{array}$ & $\begin{array}{c}\mathrm{Mg}^{2+}-\mathrm{Ca}^{2+}- \\
\mathrm{HCO}_{3}^{-}\end{array}$ \\
\hline $\mathrm{P} 17$ & $\begin{array}{c}\mathrm{Mg}^{2+}-\mathrm{Ca}^{2+}- \\
\mathrm{HCO}_{3}^{-} \\
\end{array}$ & $\begin{array}{c}\mathrm{Mg}^{2+}-\mathrm{Ca}^{2+}-\mathrm{Cl}^{-}- \\
\mathrm{HCO}_{3}^{-}\end{array}$ & $\begin{array}{l}\mathrm{Mg}^{2+}-\mathrm{Ca}^{2+}- \\
\mathrm{HCO}_{3}^{-}-\mathrm{Cl}^{-}\end{array}$ \\
\hline \multicolumn{4}{|c|}{$\mathrm{N}=$ Name of well } \\
\hline
\end{tabular}


Table 2. Water types of Valiyathodu sub-watershed

\begin{tabular}{|c|c|c|c|}
\hline $\mathbf{N}$ & $\begin{array}{c}\text { Pre } \\
\text { monsoon }\end{array}$ & Monsoon & Post monsoon \\
\hline V1 & $\begin{array}{l}\mathrm{Mg}^{2+}-\mathrm{Ca}^{2+}- \\
\mathrm{HCO}_{3}^{-}-\mathrm{Cl}^{-}\end{array}$ & $\begin{array}{l}\mathrm{Ca}^{2+}-\mathrm{Mg}^{2+}- \\
\mathrm{HCO}_{3}^{-}-\mathrm{Cl}^{-}\end{array}$ & $\begin{array}{c}\mathrm{Mg}^{2+}-\mathrm{Ca}^{2+}- \\
\mathrm{HCO}_{3}^{-}-\mathrm{Cl}^{-}- \\
\mathrm{NO}_{3}^{-}\end{array}$ \\
\hline $\mathrm{V} 2$ & $\begin{array}{l}\mathrm{Mg}^{2+}-\mathrm{Ca}^{2+}- \\
\mathrm{HCO}_{3}^{-}-\mathrm{Cl}^{-}\end{array}$ & $\begin{array}{l}\mathrm{Mg}^{2+}-\mathrm{Ca}^{2+}- \\
\mathrm{HCO}_{3}^{-}-\mathrm{Cl}^{-}\end{array}$ & $\begin{array}{l}\mathrm{Ca}^{2+}-\mathrm{Mg}^{2+}- \\
\mathrm{HCO}_{3}^{-}-\mathrm{Cl}^{-}\end{array}$ \\
\hline V3 & $\begin{array}{l}\mathrm{Mg}^{2+}-\mathrm{Ca}^{2+}- \\
\mathrm{HCO}_{3}^{-}-\mathrm{Cl}^{-}\end{array}$ & $\begin{array}{c}\mathrm{Mg}^{2+}-\mathrm{HCO}_{3}^{-}- \\
\mathrm{Cl}^{-}\end{array}$ & $\begin{array}{l}\mathrm{Mg}^{2+}-\mathrm{Ca}^{2+}- \\
\mathrm{HCO}_{3}^{-}-\mathrm{Cl}^{-}\end{array}$ \\
\hline V4 & $\begin{array}{c}\mathrm{Ca}^{2+}-\mathrm{HCO}_{3}^{-}- \\
\mathrm{Cl}^{-}\end{array}$ & $\begin{array}{c}\mathrm{Mg}^{2+}-\mathrm{Ca}^{2+}- \\
\mathrm{HCO}_{3}^{-}-\mathrm{Cl}^{-}- \\
\mathrm{NO}_{3}^{-}\end{array}$ & $\begin{array}{l}\mathrm{Mg}^{2+}-\mathrm{Ca}^{2+}- \\
\mathrm{HCO}_{3}^{-}-\mathrm{Cl}^{-}\end{array}$ \\
\hline V5 & $\begin{array}{c}\mathrm{Mg}^{2+}-\mathrm{Ca}^{2+}- \\
\mathrm{HCO}_{3}^{-}\end{array}$ & $\begin{array}{l}\mathrm{Mg}^{2+}-\mathrm{Ca}^{2+}- \\
\mathrm{HCO}_{3}^{-}-\mathrm{Cl}^{-}\end{array}$ & $\begin{array}{c}\mathrm{Mg}^{2+}-\mathrm{Ca}^{2+}- \\
\mathrm{HCO}_{3}^{-}\end{array}$ \\
\hline V6 & $\begin{array}{l}\mathrm{Mg}^{2+}-\mathrm{Ca}^{2+}- \\
\mathrm{HCO}_{3}^{-}\end{array}$ & $\begin{array}{c}\mathrm{Mg}^{2+}-\mathrm{Ca}^{2+}-\mathrm{Cl}^{-} \\
-\mathrm{HCO}_{3}^{-}\end{array}$ & $\begin{array}{l}\mathrm{Mg}^{2+}-\mathrm{Ca}^{2+}- \\
\mathrm{HCO}_{3}^{-}-\mathrm{Cl}^{-}\end{array}$ \\
\hline V7 & $\begin{array}{l}\mathrm{Mg}^{2+}-\mathrm{Ca}^{2+}- \\
\mathrm{HCO}_{3}^{-}-\mathrm{Cl}^{-}\end{array}$ & $\begin{array}{c}\mathrm{Mg}^{2+}-\mathrm{Ca}^{2+}- \\
\mathrm{HCO}_{3}^{-}\end{array}$ & $\mathrm{Mg}^{2+}-\mathrm{Ca}^{2+}$ \\
\hline V8 & $\begin{array}{l}\mathrm{Mg}^{2+}-\mathrm{Ca}^{2+}- \\
\mathrm{HCO}_{3}^{-}-\mathrm{Cl}^{-}\end{array}$ & $\begin{array}{c}\mathrm{Mg}^{2+}-\mathrm{Ca}^{2+}-\mathrm{Cl}^{-} \\
-\mathrm{HCO}_{3}^{-}\end{array}$ & $\begin{array}{l}\mathrm{Mg}^{2+}-\mathrm{Ca}^{2+}- \\
\mathrm{HCO}_{3}^{-}-\mathrm{Cl}^{-}\end{array}$ \\
\hline V9 & $\begin{array}{l}\mathrm{Mg}^{2+}-\mathrm{Ca}^{2+}- \\
\mathrm{HCO}_{3}^{-}\end{array}$ & $\begin{array}{c}\mathrm{Mg}^{2+}-\mathrm{Ca}^{2+}- \\
\mathrm{HCO}_{3}^{--}-\mathrm{SO}_{4}^{2-}\end{array}$ & $\begin{array}{c}\mathrm{Mg}^{2+}-\mathrm{Ca}^{2+}- \\
\mathrm{HCO}_{3}^{-}\end{array}$ \\
\hline V10 & $\begin{array}{l}\mathrm{Mg}^{2+}-\mathrm{Ca}^{2+}- \\
\mathrm{HCO}_{3}^{-}-\mathrm{Cl}^{-}\end{array}$ & $\begin{array}{l}\mathrm{Mg}^{2+}-\mathrm{Ca}^{2+}- \\
\mathrm{HCO}_{3}^{-}-\mathrm{Cl}^{-}\end{array}$ & $\begin{array}{c}\mathrm{Mg}^{2+}-\mathrm{Ca}^{2+}- \\
\mathrm{HCO}_{3}{ }^{-}\end{array}$ \\
\hline V11 & $\begin{array}{l}\mathrm{Mg}^{2+}-\mathrm{Ca}^{2+}- \\
\mathrm{HCO}_{3}^{-}\end{array}$ & $\begin{array}{l}\mathrm{Mg}^{2+}-\mathrm{Ca}^{2+}- \\
\mathrm{HCO}_{3}^{-}-\mathrm{Cl}^{-}\end{array}$ & $\begin{array}{c}\mathrm{Mg}^{2+} \mathrm{Ca}^{2+}-\mathrm{Cl}- \\
\mathrm{HCO}_{3}^{-}\end{array}$ \\
\hline V12 & $\begin{array}{l}\mathrm{Mg}^{2+}-\mathrm{Ca}^{2+}- \\
\mathrm{HCO}_{3}^{-}\end{array}$ & $\begin{array}{l}\mathrm{Mg}^{2+}-\mathrm{Ca}^{2+}- \\
\mathrm{HCO}_{3}^{-}\end{array}$ & $\begin{array}{c}\mathrm{Mg}^{2+}-\mathrm{Ca}^{2+}- \\
\mathrm{HCO}_{3}^{-}\end{array}$ \\
\hline V13 & $\begin{array}{l}\mathrm{Mg}^{2+}-\mathrm{Ca}^{2+}- \\
\mathrm{HCO}_{3}^{-}-\mathrm{Cl}^{-}\end{array}$ & $\begin{array}{c}\mathrm{Mg}^{2+}-\mathrm{Ca}^{2+}- \\
\mathrm{HCO}_{3}^{-}\end{array}$ & $\begin{array}{l}\mathrm{Mg}^{2+}-\mathrm{Ca}^{2+}- \\
\mathrm{HCO}_{3}^{-}-\mathrm{Cl}^{-}\end{array}$ \\
\hline V14 & $\begin{array}{l}\mathrm{Mg}^{2+}-\mathrm{Ca}^{2+}- \\
\mathrm{HCO}_{3}^{-}\end{array}$ & $\begin{array}{c}\mathrm{Mg}^{2+}-\mathrm{Ca}^{2+}-\mathrm{Cl}^{-} \\
-\mathrm{HCO}_{3}^{-}\end{array}$ & $\begin{array}{c}\mathrm{Mg}^{2+}-\mathrm{Ca}^{2+}- \\
\mathrm{HCO}_{3}^{-}\end{array}$ \\
\hline V15 & $\begin{array}{l}\mathrm{Mg}^{2+}-\mathrm{Ca}^{2+}- \\
\mathrm{HCO}_{3}^{-}-\mathrm{Cl}^{-}\end{array}$ & $\mathrm{Mg}^{2+}-\mathrm{Ca}^{2+}-\mathrm{Cl}$ & $\mathrm{Mg}^{2+}-\mathrm{Ca}^{2+}$ \\
\hline \multicolumn{4}{|c|}{$\mathrm{N}=$ Name of well } \\
\hline
\end{tabular}

In Peruvanthanam sub-watershed, during the pre monsoon season, the majority of the wells show the water type of $\mathrm{Mg}^{2+}-\mathrm{Ca}^{2+}-\mathrm{HCO}_{3}{ }^{-}, \mathrm{Mg}^{2+}-\mathrm{Ca}^{2+}-\mathrm{Cl}^{-}$and $\mathrm{Mg}^{2+}-\mathrm{Ca}^{2+}-\mathrm{HCO}_{3}^{-}-\mathrm{Cl}^{-}$which are represented in radial plots in Figs. 9, 10 and 11. From the radial plots it is revealed that the water type of the pre monsoon season is fresh or young i.e. recently recharged ground water.

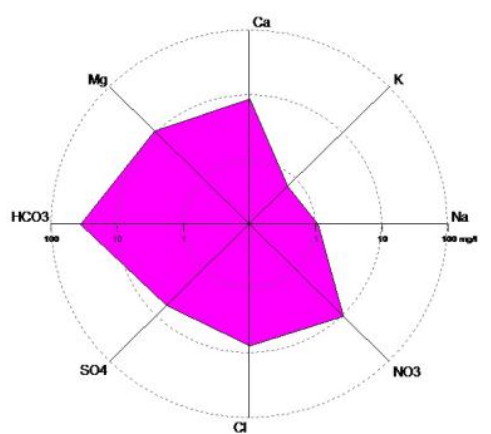

Fig. 9. $\mathrm{Mg}^{2+}-\mathrm{Ca}^{2+}-\mathrm{HCO}_{3}^{-}$water water type of Peruvanthanam sub-watershed during pre monsoon

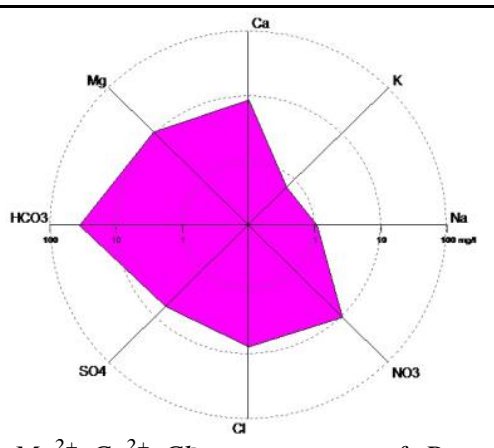

Fig. 10. $\mathrm{Mg}^{2+}-\mathrm{Ca}^{2+}-\mathrm{Cl}^{-}$water type of Peruvanthanam sub-watershed during pre monsoon

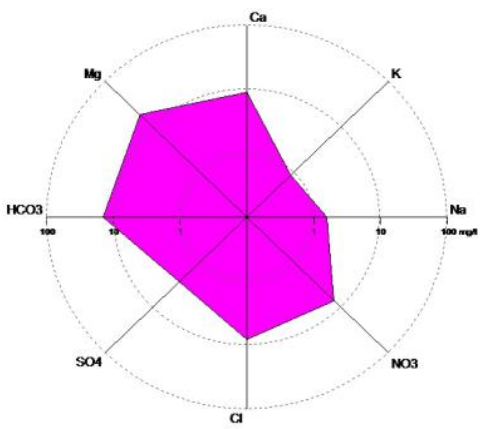

Fig. 11. $\mathrm{Mg}^{2+}-\mathrm{Ca}^{2+}-\mathrm{HCO}_{3}^{-}-\mathrm{Cl}$ type of Peruvanthanam sub-watershed during pre monsoon

The water type of the monsoon season is represented by $\mathrm{Mg}^{2+}-\mathrm{Ca}^{2+}-\mathrm{HCO}_{3}^{-}-\mathrm{Cl}^{-}, \mathrm{Mg}^{2+}-\mathrm{Ca}^{2+}-\mathrm{Cl}^{-}-$ $\mathrm{HCO}_{3}{ }^{-}$and $\mathrm{Mg}^{2+}-\mathrm{Ca}^{2+}-\mathrm{HCO}_{3}{ }^{-}-\mathrm{Cl}^{-}-\mathrm{SO}_{4}{ }^{2-}$ (Fig. 12, 13 and 14).

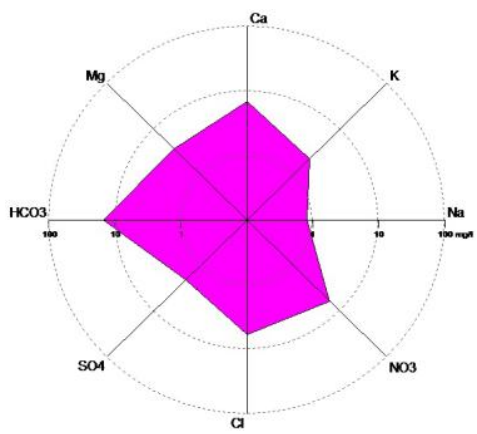

Fig. 12. $\mathrm{Mg}^{2+}-\mathrm{Ca}^{2+}-\mathrm{HCO}_{3}^{-}-\mathrm{Cl}$ water type of Peruvanthanam sub-watershed during monsoon

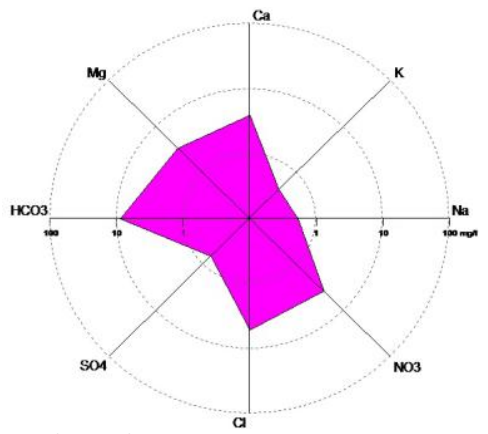

Fig. 13. $\mathrm{Mg}^{2+}-\mathrm{Ca}^{2+}-\mathrm{Cl}_{-}-\mathrm{HCO}_{3}$ water type of Peruvanthanam sub-watershed during monsoon 


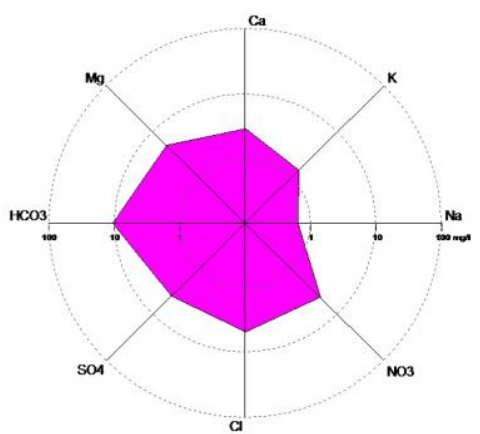

Fig. 14. $\mathrm{Mg}^{2+}-\mathrm{Ca}^{2+}-\mathrm{HCO}_{3}^{-}-\mathrm{Cl}^{-}-\mathrm{SO}_{4}{ }^{2-}$ water type of Peruvanthanam sub-watershed during monsoon

During the post monsoon season the majority of wells show $\mathrm{Mg}^{2+}-\mathrm{Ca}^{2+}-\mathrm{HCO}_{3}{ }^{-}$and $\mathrm{Mg}^{2+}-\mathrm{Ca}^{2+}-\mathrm{HCO}_{3}^{-}-$ $\mathrm{Cl}^{-}$water type in Figs 15 and 16. These figures reveal that the water type of the post monsoon season is fresh or young i.e. recently recharged.

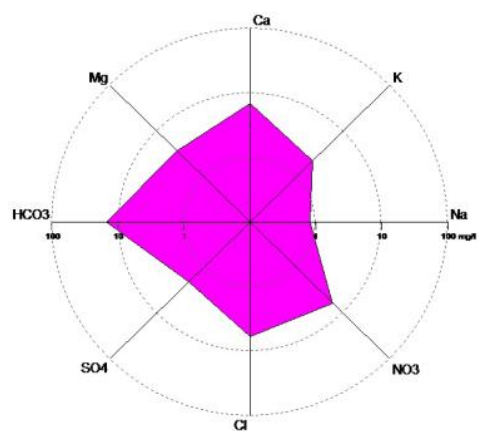

Fig. 15. $\mathrm{Mg}^{2+}-\mathrm{Ca}^{2+}-\mathrm{HCO}_{3}{ }^{-}$water type of Peruvanthanam sub-watershed during post monsoon

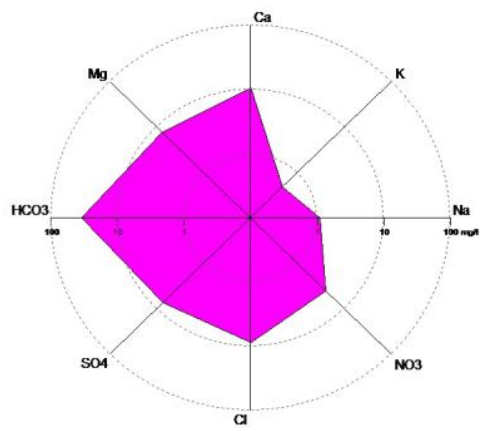

Fig. 16. $\mathrm{Mg}^{2+}-\mathrm{Ca}^{2+}-\mathrm{HCO}_{3}{ }^{-} \mathrm{Cl}^{-}$water type of Peruvanthanam sub-watershed during post monsoon

During the pre monsoon season in Valiyathodu subwatershed the majority of wells show the water type $\mathrm{Mg}^{2+}-\mathrm{Ca}^{2+}-\mathrm{HCO}_{3}-\mathrm{Cl}^{-}$and $\mathrm{Mg}^{2+}-\mathrm{Ca}^{2+}-\mathrm{HCO}_{3}-$ which is represented in radial plots in Figs 17 and 18 . Radial plots again reveal that the water type of the pre monsoon season is fresh or young i.e. recently recharged ground water and it is blended in nature. During the monsoon the majority of wells are characterized by water type $\mathrm{Mg}^{2+}-\mathrm{Ca}^{2+}-\mathrm{HCO}_{3}{ }^{-}-\mathrm{Cl}^{-}$, $\mathrm{Mg}^{2+}-\mathrm{Ca}^{2+}-\mathrm{Cl}^{-}-\mathrm{HCO}_{3}{ }^{-}$and $\mathrm{Ca}^{2+}-\mathrm{Mg}^{2+}-\mathrm{HCO}_{3}{ }^{-}-\mathrm{Cl}^{-}$(Figs 19,20 and 21) which reveal the recharge by rain water. During the post monsoon season the majority of wells are represented by the water type of $\mathrm{Mg}^{2+}$ $\mathrm{Ca}^{2+}-\mathrm{HCO}_{3}{ }^{-}-\mathrm{Cl}^{-}$and $\mathrm{Ca}^{2+}-\mathrm{Mg}^{2+}-\mathrm{HCO}_{3}{ }^{-}-\mathrm{Cl}^{-}$(Figs 22 and 23).

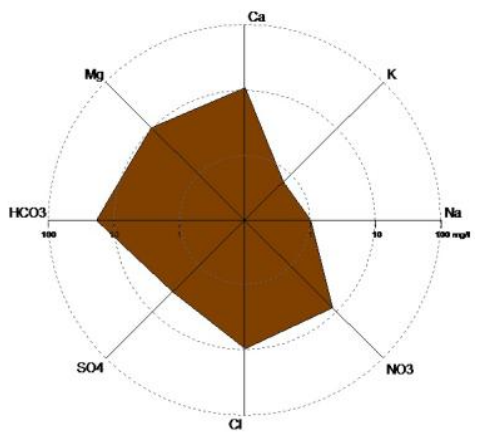

Fig. 17. $\mathrm{Mg}^{2+}-\mathrm{Ca}^{2+}-\mathrm{HCO}_{3}{ }^{-}-\mathrm{Cl}$ water type of Valiyathodu sub-watershed during pre monsoon

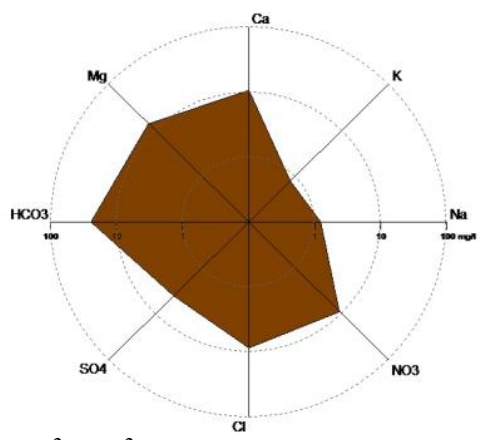

Fig. 18. $\mathrm{Mg}^{2+}-\mathrm{Ca}^{2+}-\mathrm{HCO}_{3}^{-}$water type of Valiyathodu sub-watershed during premonsoon

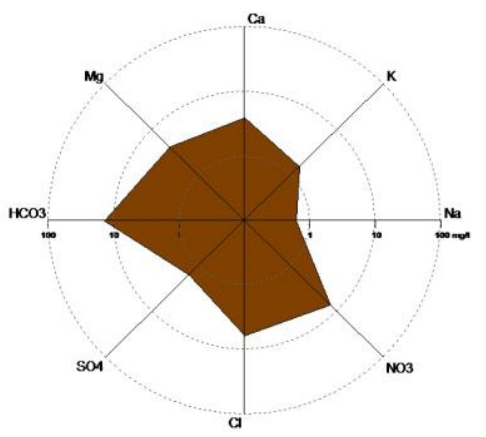

Fig. 19. $\mathrm{Mg}^{2+}-\mathrm{Ca}^{2+}-\mathrm{HCO}_{3}^{-}-\mathrm{Cl}^{-}$water type of Valiyathodu sub-watershed during monsoon

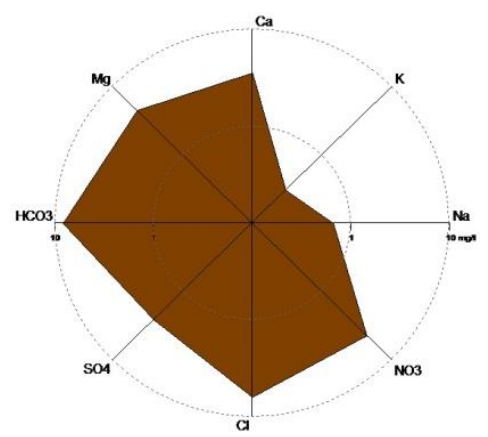

Fig. 20. $\mathrm{Mg}^{2+}-\mathrm{Ca}^{2+}-\mathrm{Cl}^{-}-\mathrm{HCO}_{3}{ }^{-}$water type of Valiyathodu sub-watershed during monsoon 


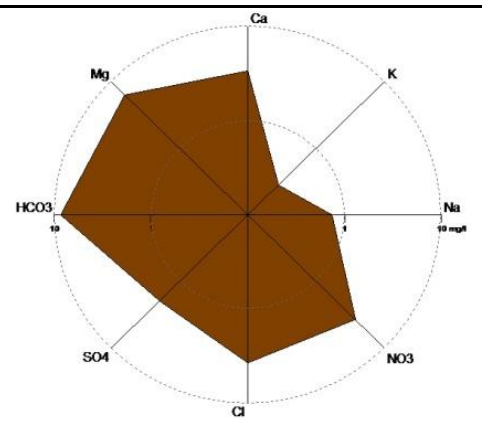

Fig. 21. $\mathrm{Ca}^{2+}-\mathrm{Mg}^{2+}-\mathrm{HCO}_{3}{ }^{-} \mathrm{Cl}^{-}$type of Valiyathodu subwatershed during monsoon

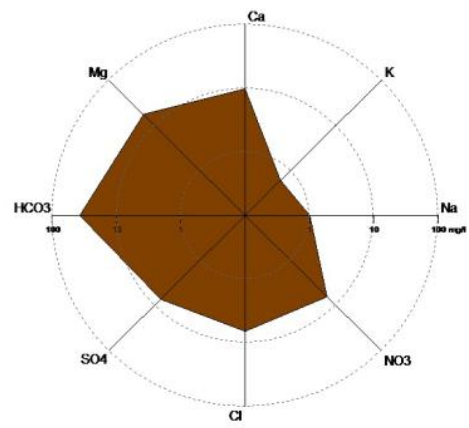

Fig. 22. $\mathrm{Mg}^{2+}-\mathrm{Ca}^{2+}-\mathrm{HCO}_{3}^{-}-\mathrm{Cl}^{-}$water type of Valiyathodu sub-watershed during post monsoon

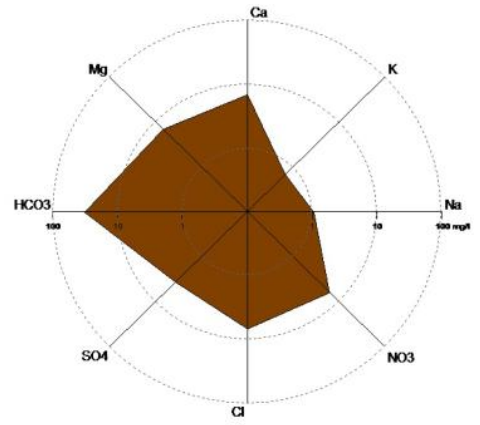

Fig. 23. $\mathrm{Ca}^{2+}-\mathrm{Mg}^{2+}-\mathrm{HCO}_{3}^{-}-\mathrm{Cl}^{-}$water type of Valiyathodu sub-watershed during post monsoon

\subsection{Stiff diagram}

Stiff diagram is a sophisticated method for demonstrating vertical changes in the chemical composition of water (Stiff 1951). The basic thing of Stiff diagram is a vertical line which has two functions. It is both depth scale of aquifers and vertical zero of axes from which the concentrations of ions are plotted on four parallel horizontal axes extending on each side. The depth of the pattern could be used as an approximate indication of the total ionic content. Cations are plotted in milli- equivalents per liter on the left side of the zero axis, and anions are plotted on the right side. Stiff diagrams can be used to visualise ionically related water from which the flow path can also be determined. If the flow path is known, it is possible to show how the ionic composition of a water body changes over space and time. It is a relatively distinctive method of showing differences or similarities in water and changes in the water composition with depth. It is useful especially for illustrating the chemical composition in hydro geologic cross sections. It can also be used for classification purposes and is useful as a symbol on a map. The stiff diagrams which represent the chemical composition of water types of Peruvanthanam subwatershed during the pre monsoon season are depicted in Figs 24, 25 and 26. Stiff diagrams reveal that $\mathrm{Mg}^{2+}-\mathrm{Ca}^{2+}-\mathrm{Cl}^{-}, \mathrm{Mg}^{2+}-\mathrm{Ca}^{2+}-\mathrm{HCO}_{3}^{-}-\mathrm{Cl}^{-}$, and $\mathrm{Mg}^{2+}$ $\mathrm{Ca}^{2+}-\mathrm{HCO}_{3}{ }^{-}$are the dominant water types of the pre monsoon season. This also agrees with the radial plots during the pre monsoon season of Peruvanthanam sub-watershed.

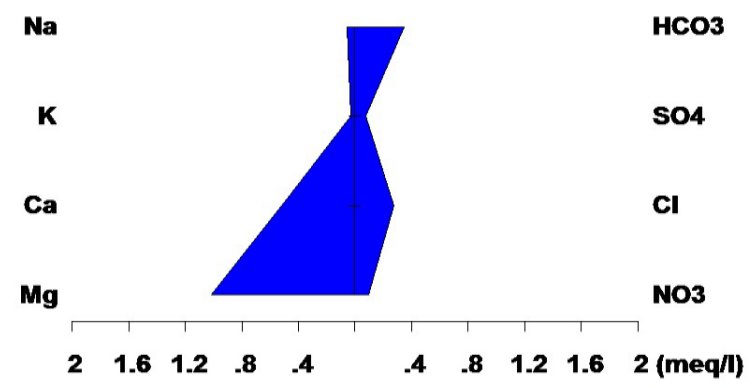

Fig. 24. Stiff diagram showing $\mathrm{Mg}^{2+}-\mathrm{Ca}^{2+}-\mathrm{Cl}^{-}$water type of Peruvanthanam sub-watershed during pre monsoon

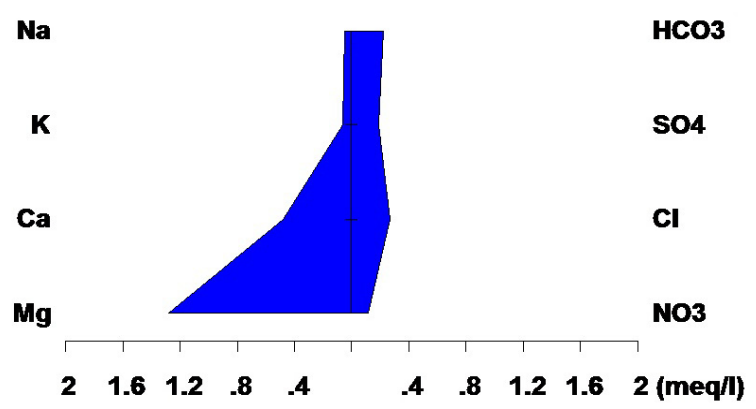

Fig. 25. Stiff diagram showing $\mathrm{Mg}^{2+}-\mathrm{Ca}^{2+}-\mathrm{HCO}_{3}{ }_{-} \mathrm{Cl}^{-}$ water type of Peruvanthanam sub-watershed during pre monsoon

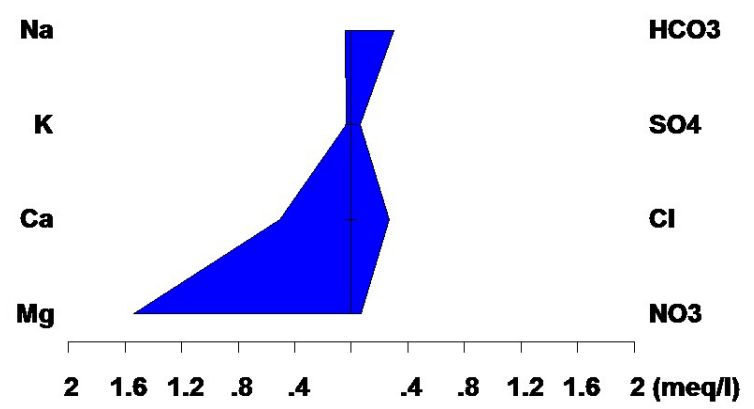

Fig. 26. Stiff diagram showing $\mathrm{Mg}^{2+}-\mathrm{Ca}^{2+}-\mathrm{HCO}_{3}^{-}$water type Peruvanthanam sub-watershed during pre monsoon

Chemical compositions of water types of Peruvanthanam sub-watershed during the monsoon season are depicted in Figs 27, 28 and 29. The diagrams reveal that $\mathrm{Mg}^{2+}-\mathrm{Ca}^{2+}-\mathrm{HCO}_{3}{ }^{-}, \mathrm{Mg}^{2+}-\mathrm{Ca}^{2+}$ $\mathrm{Cl}^{-}-\mathrm{HCO}_{3}{ }^{-}$, and $\mathrm{Mg}^{2+}-\mathrm{Ca}^{2+}-\mathrm{HCO}_{3}{ }^{-}-\mathrm{Cl}^{-}-\mathrm{SO}_{4}{ }^{2-}$ are the dominant water types of the monsoon season which is in a good agreement with the radial plots of Peruvanthanam sub-watershed during the monsoon season. 


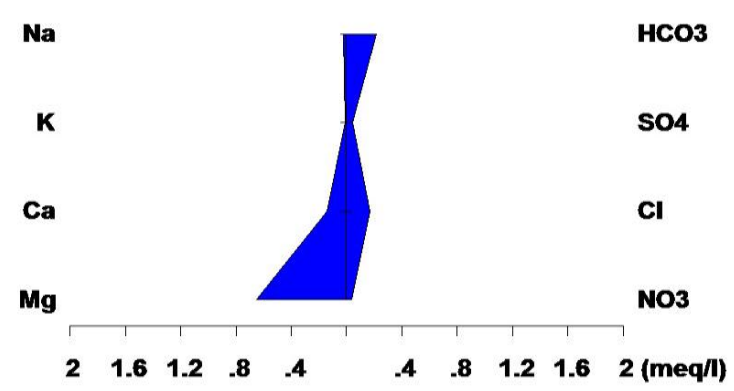

Fig. 27. Stiff diagram showing $\mathrm{Mg}^{2+}-\mathrm{Ca}^{2+}-\mathrm{HCO}_{3}^{-}$water type of Peruvanthanam sub-watershed during monsoon

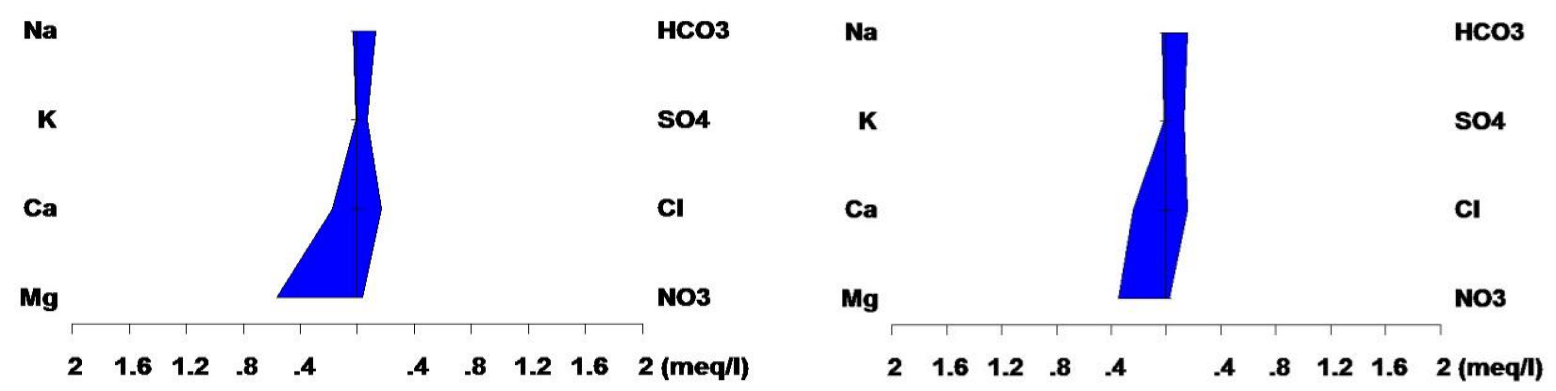

Fig. 28. Stiff diagram showing $\mathrm{Mg}^{2+}-\mathrm{Ca}^{2+}-\mathrm{Cl}_{-}^{-} \mathrm{HCO}_{3}$ water type of Peruvanthanam sub-watershed during monsoon

Fig. 29. Stiff diagram showing $\mathrm{Mg}^{2+}-\mathrm{Ca}^{2+}-\mathrm{HCO}_{3}{ }^{-} \mathrm{Cl}$ $\mathrm{SO}_{4}^{2-}$ water type of Peruvanthanam sub-watershed during monsoon

Stiff diagrams that represent chemical composition of the water types of Peruvanthanam sub-watershed during the post monsoon season are depicted in Figs 30, 31 and 32. Stiff diagrams reveal

that $\mathrm{Mg}^{2+}-\mathrm{Ca}^{2+}-\mathrm{HCO}_{3}{ }^{-}, \mathrm{Ca}^{2+}-\mathrm{Mg}^{2+}-\mathrm{HCO}_{3}{ }^{-}-\mathrm{Cl}^{-}$and $\mathrm{Mg}^{2+}-\mathrm{Ca}^{2+}-\mathrm{HCO}_{3}{ }^{-}-\mathrm{Cl}^{-}$are the dominant water types of the post monsoon season.

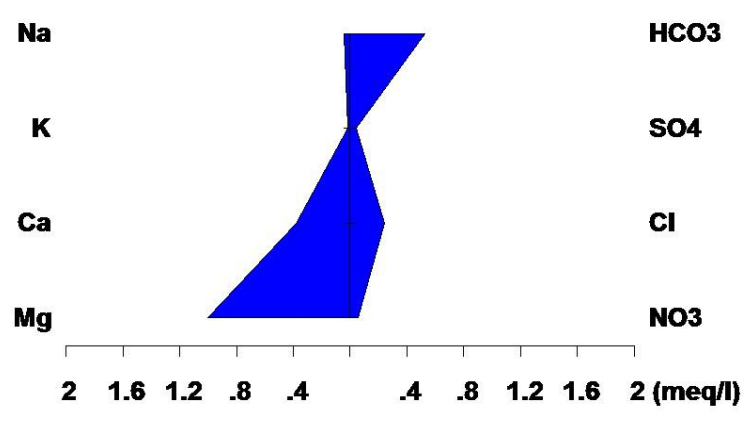

Fig. 30. Stiff diagram showing $\mathrm{Mg}^{2+}-\mathrm{Ca}^{2+}-\mathrm{HCO}_{3}$ water type of Peruvanthanam sub-watershed during monsoon

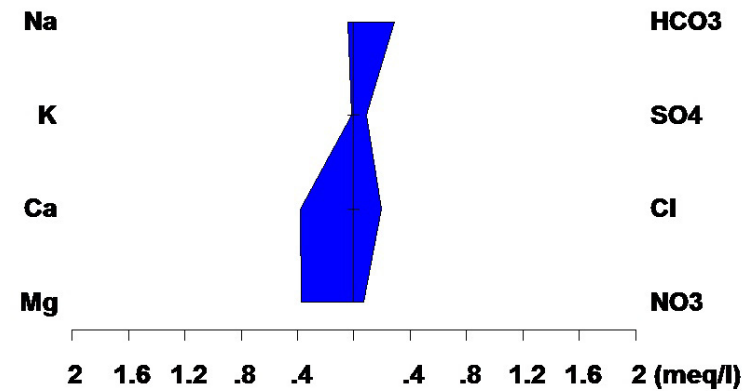

Fig. 31. Stiff diagram showing $\mathrm{Ca}^{2+}-\mathrm{Mg}^{2+}-\mathrm{HCO}_{3}{ }^{-} \mathrm{Cl}^{-}$ water type of Peruvanthanam sub-watershed during monsoon

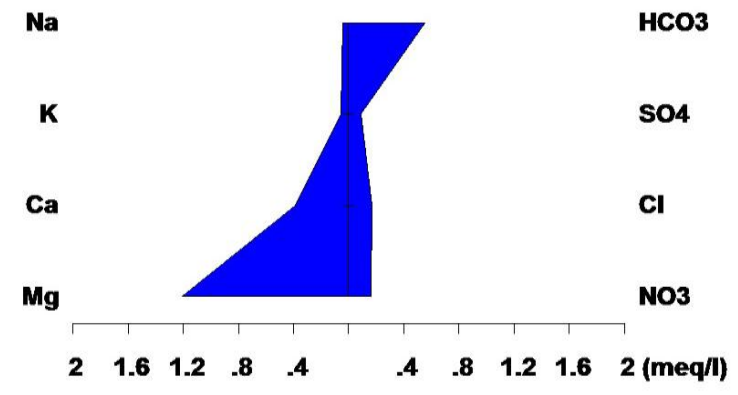

Fig. 32. Stiff diagram showing $\mathrm{Mg}^{2+}-\mathrm{Ca}^{2+}-\mathrm{HCO}_{3}{ }^{-} \mathrm{Cl}^{-}$ water type of Peruvanthanam sub-watershed during monsoon 
During the pre monsoon season in Valiyathodu sub-watershed the dominant water types are depicted in Figs 33, 34 and 35. $\mathrm{Mg}^{2+}-\mathrm{Ca}^{2+}-\mathrm{HCO}_{3}{ }^{-}-\mathrm{Cl}^{-}, \mathrm{Mg}^{2+}$ -
$\mathrm{Ca}^{2+}-\mathrm{HCO}_{3}{ }^{-}$and $\mathrm{Ca}^{2+}-\mathrm{HCO}_{3}{ }^{-}-\mathrm{Cl}^{-}$are the dominant water type.

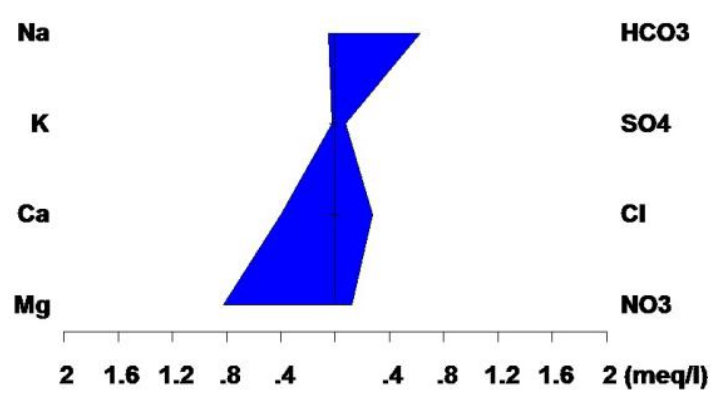

Fig. 33. Stiff diagram showing $\mathrm{Mg}^{2+}-\mathrm{Ca}^{2+}-\mathrm{HCO}_{3}{ }_{-}-\mathrm{Cl}^{-}$ water type of Valiyathodu sub-watershed during monsoon

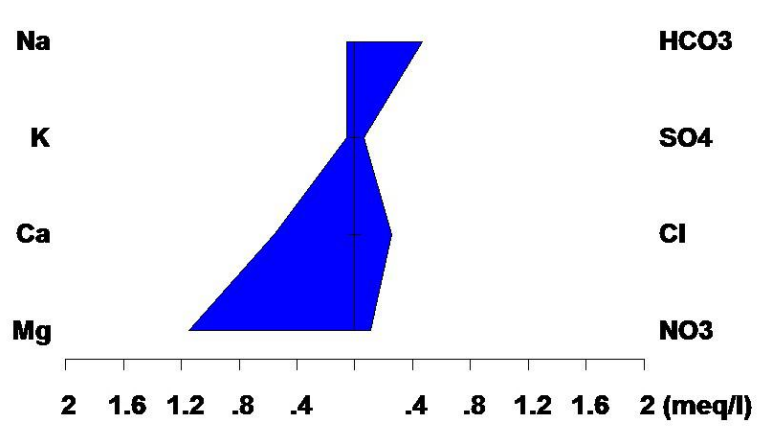

Fig. 34. Stiff diagram showing $\mathrm{Mg}^{2+}-\mathrm{Ca}^{2+}-\mathrm{HCO}_{3}^{-}$water type of Valiyathodu sub-watershed during monsoon

During the monsoon season the dominant water types of Valiyathodu sub-watershed are depicted in Figs 36, 37 and 38. $\mathrm{Mg}^{2+}-\mathrm{Ca}^{2+}-\mathrm{HCO}_{3}{ }^{-}-\mathrm{Cl}^{-}, \mathrm{Mg}^{2+}-\mathrm{Ca}^{2+}$ -

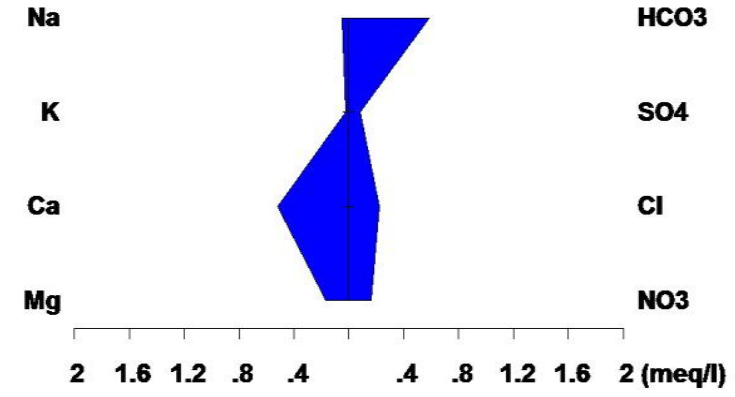

Fig. 35. Stiff diagram showing $\mathrm{Ca}^{2+}-\mathrm{HCO}_{3}^{-}{ }_{-}^{-} \mathrm{Cl}^{-}$of Valiyathodu sub-watershed during monsoon

$\mathrm{Cl}^{-}-\mathrm{HCO}_{3}{ }^{-}$and $\mathrm{Ca}^{2+}-\mathrm{Mg}^{2+}-\mathrm{HCO}_{3}{ }^{-}-\mathrm{Cl}^{-}$are the dominant water types.

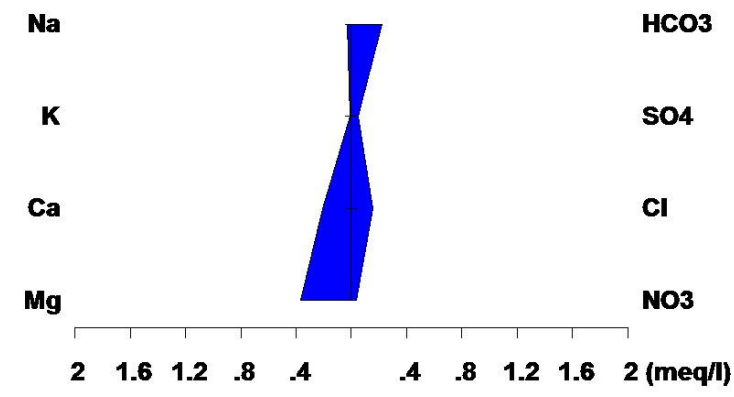

Fig. 36. Stiff diagram showing $\mathrm{Mg}^{2+}{ }_{-} \mathrm{Ca}^{2+} \mathrm{HCO}_{3}^{-} \mathrm{Cl}^{-}$ water type of Valiyathodu sub-watershed during monsoon 


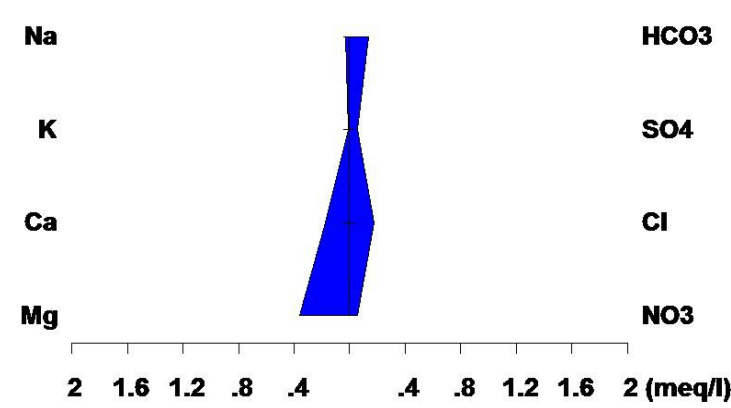

Fig. 37. Stiff diagram showing $\mathrm{Mg}^{2+}-\mathrm{Ca}^{2+}-\mathrm{Cl}^{-}-\mathrm{HCO}_{3}^{-}$ water type of Valiyathodu sub-watershed during monsoon

During the post monsoon season, dominant water types of Valiyathodu sub-watershed are depicted in Figs 39, 40 and 41. $\mathrm{Mg}^{2+}-\mathrm{Ca}^{2+}-\mathrm{HCO}_{3}{ }^{-}-\mathrm{Cl}^{-}$,

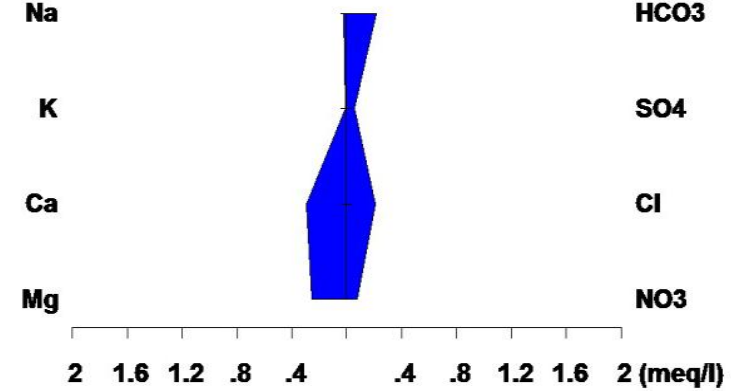

Fig. 38. Stiff diagram showing $\mathrm{Ca}^{2+}-\mathrm{Mg}^{2+}-\mathrm{HCO}_{3}{ }_{-}^{-} \mathrm{Cl}$ water type of Valiyathodu sub-watershed during monsoon

$\mathrm{Mg}^{2+}-\mathrm{Ca}^{2+}-\mathrm{HCO}_{3}{ }^{-}$and $\mathrm{Mg}^{2+}-\mathrm{Ca}^{2+}$ are the dominant water types.

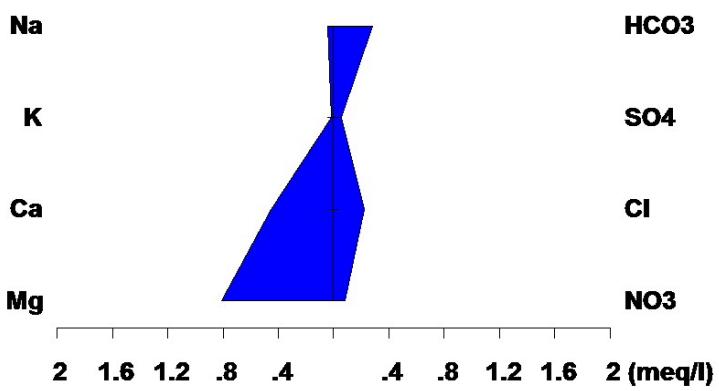

Fig. 39. Stiff diagram showing $\mathrm{Mg}^{2+}-\mathrm{Ca}^{2+}-\mathrm{HCO}_{3}^{-}-\mathrm{Cl}^{-}$water type of Valiyathodu sub-watershed during post monsoon

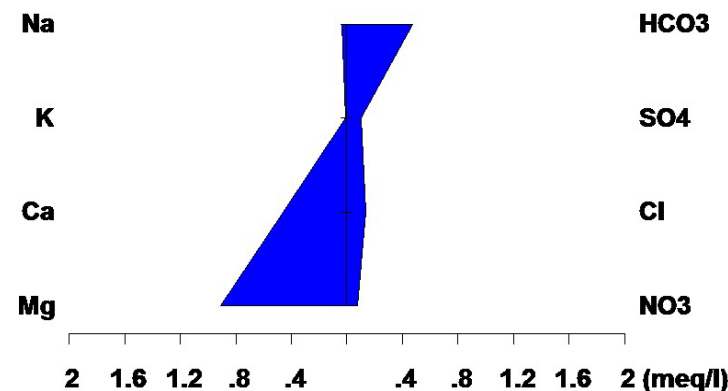

Fig. 40. Stiff diagram showing $\mathrm{Mg}^{2+}-\mathrm{Ca}^{2+}-\mathrm{HCO}_{3}{ }^{-}$water type of Valiyathodu sub-watershed during post monsoon

\section{Conclusions}

Box and Whisker diagram has revealed that the major ionic concentration of the groundwater samples of Peruvanthanam sub-watershed is $\mathrm{Mg}^{2+}>\mathrm{Ca}^{2+}>\mathrm{HCO}_{3}{ }^{-}>\mathrm{Cl}^{-}>\mathrm{SO}_{4}{ }^{2-}>\mathrm{NO}_{3}{ }^{-}>\mathrm{Na}^{+}>\mathrm{K}^{+} \quad$ and that of Valiyathodu sub-watershed is $\mathrm{Mg}^{2+}>\mathrm{Ca}^{2+}>\mathrm{HCO}_{3}{ }^{-}>\mathrm{Cl}^{-}>\mathrm{NO}_{3}{ }^{-}>\mathrm{SO}_{4}{ }^{2-}>\mathrm{Na}^{+}>\mathrm{K}^{+} . \quad \mathrm{A}$ critical analysis of Piper diagram of Peruvanthanam sub-watershed reveals that the groundwater samples of the post monsoon under zone ' $a$ ' are of prevailing bicarbonates, the samples of the pre monsoon under

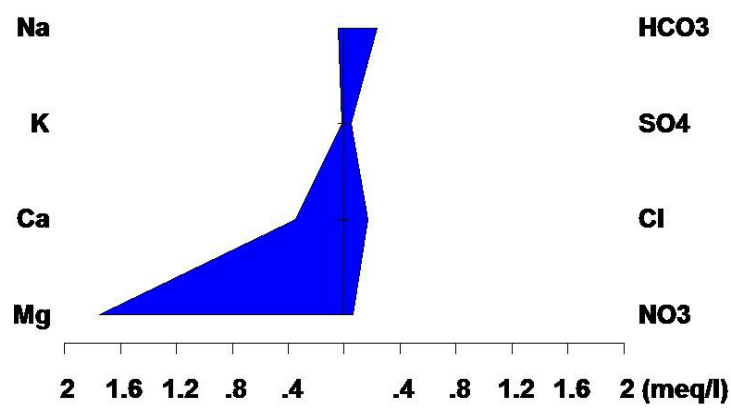

Fig. 41. Stiff diagram showing $\mathrm{Mg}^{2+}-\mathrm{Ca}^{2+}$ water type of Valiyathodu sub-watershed during post monsoon

zone ' $b$ ' are of prevailing bicarbonates, chlorides and sulphates, and those of the monsoon season under zone ' $c$ ' are of prevailing bicarbonates and chlorides. In Valiyathodu sub-watershed, the groundwater samples of the pre monsoon and post monsoon seasons under zone ' $a$ ' are of prevailing bicarbonates, the samples of the pre monsoon, monsoon and post monsoon seasons under zone ' $b$ ' are of bicarbonates, chlorides and sulphate and those of the monsoon season under 'c' zone are of prevailing bicarbonates and chlorides. Durov diagram of Peruvanthanm subwatershed depicts that in field $1 \mathrm{HCO}_{3}{ }^{-}$and $\mathrm{Ca}^{2+}$ are 
dominant which frequently indicates recharging of water in limestone, sandstone aquifers. Pre monsoon samples are dominant in field 1 . In field 2 , the water type is dominated by $\mathrm{Ca}^{2+}, \mathrm{Mg}^{2+}$ and $\mathrm{HCO}_{3}^{-}$ions. If $\mathrm{Mg}^{2+}$ in significant association with dolomite is presumed and if $\mathrm{Na}^{+}$is significant, an important ion exchange is presumed. In field $4, \mathrm{SO}_{4}{ }^{2-}$ is dominant. Post monsoon samples come under this field. In field 5 no dominants of anions or cations are observed which indicates the water exhibiting simple dissolution or mixing towards $\mathrm{Cl}^{-}$. Pre monsoon samples are dominant in this zone. In Valiyathodu sub-watershed in field $1 \mathrm{HCO}_{3}^{-}$and $\mathrm{Ca}^{2+}$ are dominant indicating that recharging of water takes place in limestone, sandstone aquifers. In field 2 the water type is dominated by $\mathrm{Mg}^{2+}$ and $\mathrm{HCO}_{3}^{-}$ions. If $\mathrm{Mg}^{2+}$ is significant, association with dolomite is presumed and if $\mathrm{Na}^{+}$is significant an important ion exchange is presumed. In field $4 \mathrm{SO}_{4}{ }^{2-}$ is dominant. In field 5 no dominance of anion or cation is observed which indicates the water exhibiting simple dissolution or mixing towards $\mathrm{Cl}^{-}$. From the radial plot and Stiff diagrams in Peruvanthanam sub-watershed, during pre monsoon, $\mathrm{Mg}^{2+}-\mathrm{Ca}^{2+}-\mathrm{HCO}_{3}{ }^{-}, \mathrm{Mg}^{2+}-\mathrm{Ca}^{2+}-\mathrm{Cl}^{-}$and $\mathrm{Mg}^{2+}-\mathrm{Ca}^{2+}-\mathrm{HCO}_{3}^{-}-\mathrm{Cl}^{-}$, during monsoon, $\mathrm{Mg}^{2+}-\mathrm{Ca}^{2+}-$ $\mathrm{HCO}_{3}^{-}-\mathrm{Cl}^{-}, \mathrm{Mg}^{2+}-\mathrm{Ca}^{2+}-\mathrm{Cl}^{-}-\mathrm{HCO}_{3}^{-}$and $\mathrm{Mg}^{2+}-\mathrm{Ca}^{2+}$ $\mathrm{HCO}_{3}{ }^{-}-\mathrm{Cl}^{-}-\mathrm{SO}_{4}{ }^{2-}$ and during post monsoon season, $\mathrm{Mg}^{2+}-\mathrm{Ca}^{2+}-\mathrm{HCO}_{3}{ }^{-}$and $\mathrm{Mg}^{2+}-\mathrm{Ca}^{2+}-\mathrm{HCO}_{3}{ }^{-}-\mathrm{Cl}^{-}$are the dominant water types. In Valiyathodu sub-watershed, during pre monsoon $\mathrm{Mg}^{2+}-\mathrm{Ca}^{2+}-\mathrm{HCO}_{3}{ }^{-}-\mathrm{Cl}^{-}$and $\mathrm{Mg}^{2+}-$ $\mathrm{Ca}^{2+}-\mathrm{HCO}_{3}^{-}$, during monsoon, $\mathrm{Mg}^{2+}-\mathrm{Ca}^{2+}-\mathrm{HCO}_{3}{ }^{-}-\mathrm{Cl}^{-}$, $\mathrm{Mg}^{2+}-\mathrm{Ca}^{2+}-\mathrm{Cl}^{-}-\mathrm{HCO}_{3}^{-}$and $\mathrm{Ca}^{2+}-\mathrm{Mg}^{2+}-\mathrm{HCO}_{3}^{-}-\mathrm{Cl}^{-}$are the dominant water types.

\section{Acknowledgements}

The authors gratefully acknowledge the Director, School of Environmental Science, Mahatma Gandhi University, Kottayam, for the support extended and facilities provided during the course of the study.

\section{References}

Ramesh, S. T, Gandhimathi, R, Nidheesh, P.V And Taywade, M. Batch and Column Operations for the Removal of Fluoride from Aqueous Solution Using Bottom Ash. Environmental Research, Engineering and Management, 2012. 2(60), P. 12-20.

Chebotarev, I. I. Metamorphism of natural waters in the crust of weathering. Geochimica et Cosmochimica Acta. 1955. 8.P. 22-28. http://dx.doi.org/10.1016/00167037(55)90015-6

Hem, J. D. 1959. Study and interpretation of the chemicalcharacteristic of natural water. USGS water supply. 1959. P. 269-271.

Back, W And Hanshaw, B. 1965. Chemical geohydrology advances in hydroscience, Academic Press. P. 49-109.

GIBBS, R.J. Mechanisms controlling world's water chemistry. Science. 1970. 170. P.1088-1090. http://dx.doi.org/10.1126/science.170.3962.1088
Srinivasamoorthy, K. 2005. Hydrogeochemistry of groundwaterin Salem district, Tamil Nadu, India; P.hD Thesis submitted to Annamalai University. P. 355-358.

Subramanian, V. 2000. Water: Quantity and Quality Perspective in South Asia, Kingston International Publishers, Surrey, United Kingdom.

Apha. 1989. Standard methods for the examination of water and waste water, American Public Health Association, Washington D C. 20. P. 2005-2605.

Williamson, J.R, Raghuraman, M.K,Cech, T.R. Monovalent cation-induced structure of telomeric DNA: the G-quartet model. Cell, 1989, (59), P. 871-880. http://dx.doi.org/10.1016/0092-8674(89)90610-7

Piper, A.M. A graphic procedure in the geochemical interpretation of water analysis. American Geophysical Union Transaction, 1944, 25, P. 914-923. http://dx.doi.org/10.1029/TR025i006p00914

Domenico, P., Schwartz, F. 1998. P. 234-247. Physical and Chemical Hydrology (second Ed.). John Wiley\& Sons Inc, New York, USA.

Back, W. 1960. Origin of hydrogeochemical facies of groundwater in the Atlantic Coastal Plain in Geochemical Cycles. P. 87-95. In Proceedings of the 21st International Geologic Congress Copenhagen, Denmark, Det Berlingske Bogtrykkeri.,

Ophori, D.U. Toth, J.. Patterns of groundwater chemistry, Ross Creek basin, Alberta,Canada. Groundwater. 1989, 27(1), P. 20-26.

Sikdar, P.K., Sarkar, S.S. And Dasgupta, S. Compositional structure of groundwater in andaround Gangajalhati, Bankuradistrict, West Bengal. Indian Journal of Earth Science. 1993, 20(1), P. 17-27.

Sikdar, P.K. And Bhattacharya, P. Geochemistry of groundwater of Jhalda-II Block, Puruliadistrict, West Bengal. Journal of Applied Hydrology . 1999, 12(2), P. 1022.

Durov, C. A. Natural waters and graphic representation of their composition. Akad, Nauk U.S.S.R, Doklady. 1948, 59, P. 87-90.

Stiff, H.A. The interpretation of chemical water analysis by means of patterns. Journal of Petroleum Technology. 1951, (3), P. 15-17.

Dr. V. B. Rekha - Department of Environmental Science, Central University of Kerala.

Main research area: Watershed management, Remote sensing and GIS and Hydrogeology

Address: Nandanam, Near. GUP. School, Painkannur. Post, Kuttippuram Via, Malappuram Dist, Pin-679590 Kerala, India

Tel.: 09846406819

E-mail: rekhavb1@gmail.com

Vice Chancellor, dr. A.V. George - Mahatma Gandhi University.

Main research area: Hydrogeology, Remote sensing and GIS, Disaster management.

Tel.: 09447285056

E-mail: aikara@sify.com

Assoc. Professor, dr. Rita Mendez - Department of Chemistry, Govt. Engineering College, Thrissur, Kerala, India.

Main research area: Water chemistry and Analytical Chemistry.

Tel.: 09446355456

E-mail: ritag@sify.com 


\title{
Lyginamoji Minimalos upės baseino aukštupio ir vidurupio pabaseinių gruntinio vandens joninė chemijos analizė
}

\author{
V. B. Rekha ${ }^{1}$, A. V. George ${ }^{2}$, M. Rita ${ }^{3}$ \\ ${ }^{l}$ Centrinis Keralos universitetas, Aplinkos mokslufakultetas, Kasaragodas, Kerala, Indija \\ ${ }^{2}$ Mahatmos Gandžio universitetas, Kotajama, Kerala, Indija \\ ${ }^{3}$ Vyriausybine inžinerijos kolegija, Chemijos fakultetas, Trisuras, Kerala, Indija
}

(gauta 2013 m. rugsèjo mèn.; atiduota spaudai 2013 m. gruodžio mèn.)

Detali gruntinio Minimalos upès baseino Peruvanthanam ir Valiyathodu pabaseinių vandens mėginiu analizè buvo atlikta naudojant AQUACHEM 4.0 programinę ịangą. Programiškai buvo ivertintos pagrindinės cheminės charakteristikos ir suprasti geocheminiai vandens procesai. Studija parode, jog katijonai: kaip natris $\left(\mathrm{Na}^{+}\right)$, kalis $\left(\mathrm{K}^{+}\right)$, kalcis $\left(\mathrm{Ca}^{2+}\right)$ ir magnis $\left(\mathrm{Mg}^{2+}\right)$, anijonai: hidrokarbonatas $\left(\mathrm{HCO}_{3}{ }^{-}\right)$, sulfatas $\left(\mathrm{SO}_{4}{ }^{2-}\right)$, chloridas $\left(\mathrm{Cl}^{-}\right)$, nitratas $\left(\mathrm{NO}_{3}{ }^{-}\right)$, yra esminiai tiriamuosiuose vandenyse. Nustatymo metodai: a) děžès ir Whisker diagrama, b) Piper diagrama, c) Durov diagrama, d) Radialinis plotas ir e) Stiff schemos, buvo taikomi sezoninei cheminių medžiagų variacijai vandenyse pavaizduoti. Pagrindine gruntinio Peruvanthanam pabaseinio vandens méginiuose jonu koncentracija buvo $\mathrm{Mg}^{2+}>\mathrm{Ca}^{2+}>\mathrm{HCO}_{3}{ }^{-}>\mathrm{Cl}^{-}>\mathrm{SO}_{4}{ }^{2-}>\mathrm{NO}_{3}{ }^{-}>\mathrm{Na}^{+}>\mathrm{K}^{+}$, o Valiyathodu - $\mathrm{Mg}^{2+}>\mathrm{Ca}^{2+}>\mathrm{HCO}_{3}^{-}>\mathrm{Cl}^{-}>\mathrm{NO}_{3}^{-}>\mathrm{SO}_{4}{ }^{2-}>\mathrm{Na}^{+}>\mathrm{K}^{+}$. Analizuojant kritinę Piper diagramą buvo nustatyta, jog Peruvanthanam upès pabaseinio rajone iki lietingojo sezono vandens mėginiai buvo sodrūs hidrokarbonatais, chloridais, sulfatais. Lietinguoju sezonu mèginiuose buvo didesnès hidrokarbonatu ir chloridu koncentracijos, o po lietingojo sezono mėginiuose vyravo hidrokarbonatai. Valiyathodu pabaseinio mèginiuose prieš lietingaji sezoną ir po jo dominavo hidrokarbonatinè sudètis, o lietinguoju sezonu vyravo abi jonu rūšys: tiek chloridai, tiek hidrokarbonatai. Radialiniais plotais ir Stiff diagramomis Peruvanthanam pabaseinio méginiuose buvo nustatyta, jog $\mathrm{Mg}^{2+}-\mathrm{Ca}^{2+}-\mathrm{HCO}_{3}^{-}, \mathrm{Mg}^{2+}-\mathrm{Ca}^{2+}-\mathrm{Cl}^{-}$ir $\mathrm{Mg}^{2+}-\mathrm{Ca}^{2+}-\mathrm{HCO}_{3}{ }^{-} \mathrm{Cl}$ yra dominuojantys vandens tipai ne lietinguoju metu. $\mathrm{Mg}^{2+}-\mathrm{Ca}^{2+}-\mathrm{HCO}_{3}{ }^{-}-\mathrm{Cl}^{-}, \mathrm{Mg}^{2+}-\mathrm{Ca}^{2+}-\mathrm{Cl}^{-}-\mathrm{HCO}_{3}{ }^{-}$ir $\mathrm{Mg}^{2+}-\mathrm{Ca}^{2+}-$ $\mathrm{HCO}_{3}{ }^{-}-\mathrm{Cl}^{-}-\mathrm{SO}_{4}{ }^{2-}$ yra vyraujantys jonai lietinguoju metu, o $\mathrm{Mg}^{2+}-\mathrm{Ca}^{2+}-\mathrm{HCO}_{3}{ }^{-}$ir $\mathrm{Mg}^{2+}-\mathrm{Ca}^{2+}-$ $\mathrm{HCO}_{3}{ }^{-}$-Clyra dominuojantys praejjus lietingajam sezonui. Valiyathodu pabaseinyje nelietinguoju sezonu dominuoja šie vandens tipai: $\mathrm{Mg}^{2+}-\mathrm{Ca}^{2+}-\mathrm{HCO}_{3}{ }^{-}-\mathrm{Cl}^{-}$ir $\mathrm{Mg}^{2+}-\mathrm{Ca}^{2+}-\mathrm{HCO}_{3}^{-}$, liūties sezonu šie: $\mathrm{Mg}^{2+}-\mathrm{Ca}^{2+}-\mathrm{HCO}_{3}^{-}-\mathrm{Cl}^{-}, \quad \mathrm{Mg}^{2+}-\mathrm{Ca}^{2+}-\mathrm{Cl}^{-}-\mathrm{HCO}_{3}^{-}$ir $\mathrm{Ca}^{2+}-\mathrm{Mg}^{2+}-\mathrm{HCO}_{3}^{-}-\mathrm{Cl}^{-}$, o praejus lietingajam laikotarpiui šie: $\mathrm{Mg}^{2+}-\mathrm{Ca}^{2+}-\mathrm{HCO}_{3}{ }^{-}-\mathrm{Cl}^{-}$ir $\mathrm{Ca}^{2+}-\mathrm{Mg}^{2+}-\mathrm{HCO}_{3}^{-}-\mathrm{Cl}^{-}$. 